\title{
La régénération des ovaires chez Polycelis nigra [Ehrenb.].
}

\author{
Par \\ Jules Zweibaum \\ (Modena).
}

Avec Planches IX et X.

Eingegangen am 11. Juli 1914.

Table des matières.

III. La régénération des ovaires (germigène et vitellogène) dans les conditions normales . . . . . . . . . . . . . . . . . . . . . 434

IV. L'influence de la lumière . . . . . . . . . . . . . . . . . . . . . 445

V. L'influence de la température . . . . . . . . . . . . . . . . . . . 447

VI. L'influence du milieu salin . . . . . . . . . . . . . . . . . . . . 451

VII. La régénération répétée des ovaires . . . . . . . . . . . . . . . 462

VIII. Conclusions . . . . . . . . . . . . . . . . . . . . . . . . . . . 464

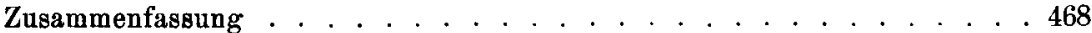

Bibliographie .. . . . . . . . . . . . . . . . 468

Explication des planches. . . . . . . . . . . . . . . . . . 470

\section{Introduction.}

De tous les travaux sur la régénération faits jusqu'à nos jours, une minime partie s'est occupée des phénomènes intimes, histologiques s'accomplissant durant la régénération, la plus grande partie s'est occupée des phénomènes externes dans le sens le plus large.

Quelques données histologiques ont été acquises avec certitude par les recherches de Morgan, Nussbaum, Schultz, Lang et d'autres, mais ces données concernent surtout les questions relatives à l'histogénèse somatique - les questions relatives à l'histogénèse germinale ont été presque complètement ignorées.

Cette dernière pourtant présente un intérêt tout à fait particulier pour la Biologie si l'on considère les idées actuellement 
régnantes sur la spécificité cellulaire défendues par BARD et sa célèbre formule »Omnis cellula e cellula ejusdem naturae « et la théorie de la continuité du plasma germinatif de Weismans. En effet comme il a été demontré par Boveri pour Ascaris megalocephala - il semble que les cellules germinales différent essentiellement des cellules somatiques par la quantité de chromatine.

Il est évident que la question de l'origine des éléments germinaux prend une importance capitale. Les éléments sexuels peuventils être régénérés et aux dépens de quoi?

Or les expériences faites sur les animaux supérieurs sont en accord avec la théorie de Weismann. Ce n'est pas le cas de citer les expériences de Pugnat sur la régénération d'une partie de l'ovaire chez le lapin - aussi que les expériences de Lo'throp sur le même animal. Les expériences de Levy sur Bufo vulgaris pourraient avoir une valeur importante si l'on avait la certitude que l'auteur a enlevé complètement les ovaires, mais l'auteur lui même n'est pas sûr à ce point.

Pour les animaux inférieurs les auteurs sont arrivés à des conclusions très peu concordantes - et dans plusieurs cas décidément négatives.

Chez les hydres on a observé la régénération des éléments sexuels - ainsi chez les Tubularies Driesch a trouvé la régénération des gonades. Mais ces cas nous intéressent relativement peu si nous considérons - que ces animaux penvent se reproduire aussi agamiquement. Parmi les Echinodermes on a demontré la possibilité de régénération des éléments sexuels chez une holoturie (Tyone fusa). KING l'a observé aussi chez les étoiles de mer. Au contraire certaines autres holoturies ${ }^{1)}$ reforment en quelques jours - l'appareil digestif, leur poumon gauche avec les vaisseaux, mais leurs organes génitaux ne se reproduisent pas.

Chez les autres invertébrés les tentatives de régénération des organes sexuels ont conduit toujours à des résultats négatifs.

Ainsi Davydoff - qui a étudié les Némertiens trouve que chez Cerebratulus tous les organes peuvent être régénérées sauf les gonades.

Selon Nussbaum - le Lombriciens ne régénèrent pas les éléments sexuels.

Il est difficile d'interpréter les résultats obtenus par SchuLtz et aussi par R. Montr pour les Triclades marines, si l'on considère la

1) Y. Delage, La structure du protoplasma etc. p. 94. 
disposition particulière des ovaires chez ces animaux. Et même chez les planaires d'ean douce qui sont dévenues classiques pour l'étude de la régénération, les résultats, en ce qui concerne les ovaires, ont été négatifs.

LEhNer'T même en étudiant la régénération de l'ovaire dit que l'on ne pouvait espérer autrement les planaires régénérées ne montrent pas de traces des organes sexuels. La constatation de Morgan pour Planaria lugubris est trop isolée, pour qu'on puisse lui attribuer une valeur décisive, tant plus si l'on considère les résultats négatifs obtenus par d'autres auteurs qui ont étudié la régénération de cet animal.

Pour la gonade masculine les recherches ont été plus favorisées. Il semble précisement que $P l$. lugubris régénère les testicules aux dépens des éléments somatiques. Schultz en effet dans son travail sur la régénération a pu constater et démontrer l'histogénèse des testicules chez cette planaire - mais il n'a pas réussi de trouver l'ovaire régénéré.

Dernièrement A. Mon'ti a démontré la possibilité de la régénération des ovaires chez $P l$. torva - sans pouvoir démontrer leur origine. Et e'est le seul cas que l'on connait.

Il n'existe pas à ma mémoire des travaux histologiques sur la régénération de l'ovaire.

La question done est toujours ouverte.

Parmi les travaux qui traitent les questions relatives aux influences externes favorisant la régénération des ovaires, nous devons citer Berninger. Cet auteur en effet démontre l'action nuisible du jeûne sur la vie et sur le développement des oocytes.

On connait très peu de choses sur les autres conditions favorisant la régénération en générale et rien du tont sur ce qui concerne en particulier l'ovaire: la température - la lumière, le milieu salin. Parmi les auteurs qui ont étudié ces derniers facteurs - il faut citer Stockard et aussi Morgulss. Mais ces auteurs traitent la question d'ordre général et on n'a jamais étudié les facteurs spécifiques de la régénération de l'ovaire. Cette question pourtant peut présenter un intérêt particulier non seulement d'ordre thèorique, mais aussi pratique élévé.

Voilà les buts principaux de mon travail, voilà les arguments que j'ai cherchés de traiter en entreprenant cette recherche. 


\section{Méthode et technique.}

La nécessité d'avoir un grand nombre d'individus ainsi que le fait que Polycelis nigra a été très peu étudié au point de vue de la régénération - m'ont induit à m'arrêter sur cette espèce.

En effet Polycelis nigra est très abondant dans les eaux des environs de Modène, et je pouvais très facilement me procurer des centaines d'individus. Pl. torva, Pl. lugubris et Dendrocoelum lacteum s'y trouvent aussi, mais en très petit nombre. Polycelis nigra qui m'ont servi pour les expériences mésurent à l'état de l'extension normale de 8 à $9 \mathrm{~mm}$. de longueur sur $1,5 \mathrm{~mm}$. de largeur.

Les ovaires de Pol. nigra se trouvent immédiatement au dessous du ganglion cérébral entre la $2^{\mathrm{e}}$ et la $3^{\mathrm{e}}$ ramification intestinale.

Il est facile de couper les individus an nivean voulu en procédant de la manière suivante: Au dessous du verre de l'opération on colle le carton, divisé en certain nombre de cercles concentriques, espacés, l'un de l'autre d'un millimètre. Moyennant une coupe on sépare un segment de 3 à 4 et même de $2 \mathrm{~mm}$. suffisant pour enlever complètement les ovaires, en laissant à régénérer la portion caudale de 5 à $6 \mathrm{~mm}$. En plusieurs reprises je me suis assuré d'avoir enlevé complètement les ovaires, arec ces systèmes de coupes.

Les individus ainsi coupés ont été placés dans des vases de $5 \mathrm{~cm}$. de diamètre sur $4 \mathrm{~cm}$. de hauteur, remplis d'ean potable en y ajoutant quelques Lemna. Les liquides de ces cultures ont été changés tous les 2 on 3 jours.

Les Polycelis ont été nourris avec des lombriciens. Les individus opérés pourtant, ne prennent pas de nourriture qu'après la régénération complète de tous les organes. Dans mes expériences cette régénération avait lieu en 15 jours après l'opération, à la température de $14^{\circ}$ à $16^{\circ} \mathrm{C}$.

C'est la température du laboratoire, où j'ai tenu toutes mes cultures.

Les divers agents employés sont le jeûne, la nutrition abondante, la lumière, la température. Parmi les agents d'ordre chimique j'ai employé le $\mathrm{NaCl}$, le $\mathrm{CaCl}_{2}$ et le $\mathrm{FeCl}_{3}$, aussi que le $\mathrm{HgCl}_{2}$ et $\mathrm{As}_{4} \mathrm{O}_{6}$.

Pour étudier l'influence de l'alcalinité et de l'acidité du milieu j'employais le $\mathrm{NaOH}$ et $\mathrm{HCl}$.

En fixant les animaux tous les jours, je pouvais établir avec exatitude le temps nécessaire pour la formation des divers organes, aussi qu'établir l'histogénèse de l'ovaire. 
Il me reste à dire quelques mots sur la méthode de la fixation. Un des meilleurs fixatifs des tissus, aussi pour l'extension de l'animal, est le liquide de Zenker un peu modifié: au lieu du liquide de MülLER, j'ai employé le bichromate de potassium à $2,5 \%$ et sur $100 \mathrm{~cm} .{ }^{3}$ de ce liquide j'ai ajouté $7 \mathrm{gr}$. de sublimé et $5 \mathrm{~cm} .^{3}$ d'acide acétique glacial. Avec cette méthode on obtient des fixations absolument bonnes pour les éléments parenchymateux - surtout lorsque ce liquide est employé à $50^{\circ}--60^{\circ} \mathrm{C}$. La fixation ne doit pas durer plus que 5 minates. Après un court lavage dans l'eau distillé on passe très graduellement dans les alcools, où le séjour ne doit pas être supérieur à 10-15 min. Dans l'alcool et le xilol les pièces doivent être tenues 10 min. et dans le xilol pur pas plus de 15 min. On passe les pièces dans la paraffine et le xilol pendant $15 \mathrm{~min}$. et enfin dans la paraffine à $50^{\circ}$, et pas plus dure, pendant $20 \mathrm{~min}$. Un séjour plus prolongé dans la paraffine rend les pièces très fragiles. La coloration à été faite sur les coupes. J'ai eu les meilleurs colorations arec le carmin boracique et le vert de méthyle. Les éléments parenchymateux se colorent en vert clair, les oocytes en rouge.

Une autre coloration que j'ai employé avec de très bons résultats est la double, aussi avec l'orange $G$ et l'hématéine $I A$. Les éléments parenchymateux se colorent en bran-violet, les occytes en jaune foncé.

Les autres colorations seront indiquées à part.

\section{La régénération des ovaires dans les conditions normales.}

Avant d'entrer dans la question il est nécessaire de rappeler la disposition et la structure des ovaires chez Polycelis nigra.

Les germigènes unilobés sont situés dans la partie antérieure du corps, presque immédiatement au dessous du ganglion cérébral entre la deuxième et la troisième ramification intestinale. Ils sont placés vers la face ventrale de l'animal et dans le voisinage immédiat des troncs nerveux longitudinaux (Pl. IX Fig. 1-2).

Immédiatement au dessus du germigène se trouvent les vitellogènes unibolés, lesquels quelques fois se montrent plurilobés (Pl. X Fig. 15 et $16, n t)$.

Le germigène est entouré d'une membrane anhyste, tunica propria. Pourtant dans le voisinage immédiat de l'oviducte on trouve de deux à trois noyaux ovales, très petits. Ces noyaux se trouvent uniquement en conctat avec l'oviducte, tout le reste de la membrane 
en est complètement privé. Le vitellogène chez Pol. nigra est entouré d'une membrane uniformément anhyste.

Les oviductes tapissés par épithélitim courent le long des troncs nerveux latéraux.

Je dois m'arrêter quelques instants sur le parenchyme à fin de pouvoir avec majeure elairté démontrer l'histogénèse de l'ovaire. Le parenchyme est constitué, comme on le sait, essentiellement de deux catégories d'éléments cellulaires.

Dans la première catégorie nous mettons les cellules à un ou plusieurs prolongements, avec les noyaux ovales et plus rarement ronds contenant plusieurs granulations chromatiques (Pl. IX Fig. 4, 5, 7). Chez Pol. nigra ces cellules à l'état normale constituent les éléments prédominants dans le parenchyme et elles sont surtout abondantes dans la partie antérieure du corps au dessus et au dessous du ganglion cérébral. On les observe aussi en plus petit nombre parmi les ramifications intestinales. Ce sont les cellules de soutiens. Avec leurs prolongements elles s'unissent aux autres cellules en déterminant la formation d'un réticulé aux mailles très fines. Chez les individus normanx on trouve desseminees dans le parenchyme entre les cellules du premier type, les cellules libres d'une forme irrégulière, avec un gros noyau central sans aucune granulation chromatique, lequel se colore en brun foncé avec l'orange $G$ et l'hématéine I A. Le protoplasma de ces cellules se colore en jaune et se montre parfaitement homogène ou rarement très finement granulé. Ces cellules sont plus rares chez les individus normaux que les cellules du premier type. Ce sont les cellules embryonnaires.

Nous allons traiter successivement de la régénération du germigène et ensuite de celle du vitellogène.

Germigène. Déjà quelques heures après l'opération on observe une très forte prolifération de ces cellules embryonnaires avec les nombreuses mitoses dans tous les stades; chez les individus normanx on trouve quelquefois aussi les mitoses dans ces cellules, comme il a été observé aussi par LANG chez Pl. polychroa. Je ne pouvais trouver des mitoses dans les autres cellules somatiques de Pol. nigra dans mes très nombreuses préparations, même malgré un examen très scrupuleux. J'ai observé les individus dès deux heures après l'opération jusqu'à $14 \mathrm{~h}$. tous les 4 heures et plus tard tous les jours sans pouvoir jamais trouver une seule mitose dans ces éléments. Au contraire, 4 heures après l'opération, on observe dans une seule sec- 
tion une quantité de mitoses dans les cellules embryonnaires. A 7 heures après l'opération on observe une accumulation très forte de cellules embryonnaires dans la partie antérieure du corps. Parmi ces cellules on distingue certaines différences qui consistent uniquement dans la plus ou moins faible granulation du protoplasma. Parfois les noyaux de ces cellules montrent avec des forts grossissements quelques granulations chromatiques. Ce sont les cellules de régénération (Pl. IX Fig. 4, 5 etc., $c r$ ). Selon leur position et par l'ensemble des conditions réalisées par les divers organes, ces cellules prennent

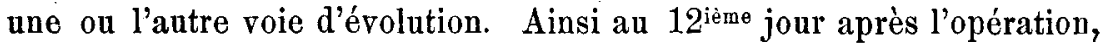
dans la position normale des ovaires, c. à d. au dessous du ganglion nerveux et dans le voisinage proche des troncs nerveux longitudinaux, on observe le grand nombre de cellules de régénération que nous

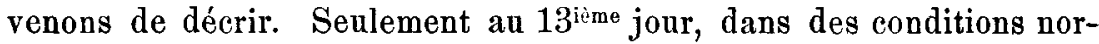
males et à la température de $14^{\circ}$ à $16^{\circ} \mathrm{C}$, , on voit parmi ces cellules une seule se différencier in situ done dans la position normale des ovaires, et entrer en évolution (Pl. IX Fig. 4-5, (m).

Cette différenciation dans les conditions exposées plus hant, s'accomplit toujours après la régénération de tous les autres systèmes organiques: du système nerveux, de l'intestin, des testicules et de l'oviducte. Déjà Lavg a observé chez $P l$. polychroa la régénération des oviductes, mais cet auteur n'a pas réussi à constater la régénération des ovaires.

Cette cellule embryonnaire ainsi différenciée augmente de volume tout en conservant sa forme irregulière. Son noyau se charge profondément de chromatine et se colore très intensément en vert par la double coloration du vert de méthyle et carmin boracique ou en brun-violet par la double arec l'orange $G$ et l'hématéine I A. Le cytoplasma de cette cellule se colore respectivement en rouge et en jaune-brun. Elle se charge de fines granulations. Le noyau sphérique ou ovale est entouré d'une couche de cytoplasma hyalin périnucléaire. Cette cellule qui au debut de son développement ne différait guère des cellules embryonnaires se montre à ce stade ainsi différenciée et elle constitue la cellule mère de l'ovaire. Elle est entourée par des cellules de régénération plus pétites, moins granuleuses, très souvent parfaitement homogènes et aux noyaux moins chromatiques. Ces cellules ne prennent nullement part dans la régénération des ovaires.

$\mathrm{Au}$ stade immédiatement successif cette cellule entre en évolution. Son noyau se divise pour la première fois mitotiquement en 
donnant lieu à deux cellules filles (Pl. IX Fig. 6, ov). Le plan de division est parallèle à l'axe longitudinal du future ovaire. Les denx cellules se disposent parallèlement à la parois du corps. Plus tard cette position change et précisément les deux cellules se disposent une vers la branche verticale de l'intestin, l'autre vers l'extérieur c. à. d. vers la paroi du corps (Pl. IX Fig. 7, ovi, ove). Ce sont les premières ovogonies du prochain ovaire. Ces cellules grandissent toujours inégalement et précisement, dans tous les cas observés, c'est toujours la cellule interne qui devient plus grande tandis que l'autre, qui reste vers l'extérieur, est plus petite. Leurs protoplasmas deviennent plus granuleux encore et elles se distinguent immédiatement des cellules de régénération qui les entourent. Au stade immédiatement snccessif, chose étrange, dans les nombreux cas observés, j'ai constaté la mitose uniquement dans la cellule plus grande tandis que l'autre plas petite était en repos (Pl. IX Fig. 8 et 9). Ce fait est constant. C'est la cellule la plus grande qui entre la première en évolution. Son noyau se divise mitotiquement et donne lieu à deux cellules. Le germigène se montre alors tricellulaire (Pl. X Fig. 10-11, ov).

A ce stade on n'observe pas encore la membrane enveloppant l'ovaire, ni l'accumulation du pigment brun-noir caractéristique de l'ovaire constitué. Ces cellules sont entourées d'autres cellules parenchymateuses et les trois ovogonies entrent en évolution ultérieure. Je ne pouvais malgré les nombreuses préparations saisir le moment de la division de ces premières ovogonies et toujours je trouvais déjà le germigène contenant quatre ovogonies disposées dans le même plan (Pl. X Fig. 12,ov). Ici donc le plan de la division est le même que dans le premier cas. C'est seulement dans ce stade tétracellulaire qu'on observe quelquefois la première formation de tunica propria, très mince encore et sur quelques points les premières granules pigmentaires. Plus tard encore cette membrane s'épaissit au fur et à mesure de la formation des nouvelles ovogonies. La multiplication de ces quatre ovogonies poursuit. Il est possible alors de trouver tous les stades intermédiaires jusqu'à la formation de 64 ovogonies; et les plans de la division deviennent perpendiculaires à l'axe longitudinal de l'ovaire. Dans une coupe transversale de l'ovaire, à ce stade, on en observe plusieurs disposées tont antour de la paroi interne de l'ovaire (Pl. X Fig. 13). Ces cellnles ont la forme aplatie avec le noyaa allongé et ne différent guère dans leur structure des ovogonies.

Cette disposition donne l'aspect d'une enveloppe cellulaire comme 
il a été observé par Moselex et Kennel, mais déjà JiJna avec raison, et aussi Woopworth, ont nié l'existance de cette membrane chez les Triclades d'eau douce.

Plus tard, lorsque le germigène contient les nombreux oocytes, sa membrane montre quelques noyaux en contact avec l'oviducte; tout le reste de la membrane est complètement privé des éléments nucléaires.

Dans ce stade lovaire prend la forme normale, celle d'une poire, et en s'allongeant il arrive à se réunir avec l'oviducte, qui est déjà complètement constitué.

Cette évolution de l'ovaire s'accomplit, dans les conditions expliquées plus haut, au $16^{\text {ième }}$ jour, c. à d. en 5 jours après la première différenciation da la cellule - mère de l'ovaire. L'ovaire se montre enveloppé de tunica propria plus ou moins mince et sur son pourtour on observe une assez forte déposition de pigment.

Jasqu'au stade de 32 ovogonies on n'observe pas des nucléoles et la vésicule germinative ne montre aucune structure appréciable. C'est seulement à partir de ce stade qu'il m'étais possible de trouver un gros nucléole bien distinet du noyau, moyennant la coloration double d'orange $\mathrm{G}$ et d'bématéine I A. (Pl. X Fig. 13, nuc). Généralement l'évolution de l'ovaire gauche et droit se fait parallèlement, aussi que la première différenciation de la cellule embryonnaire.

Ainsi finit le période de multiplication des ovogonies et commence celui de l'accroissement. Les ovogonies, au moins une partie d'elles, deviennent plus grandes; leurs noyaux perdent la propriété

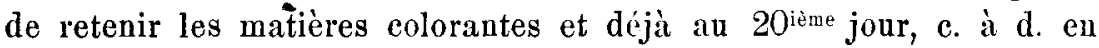
9 jours après la première différenciation de la cellulemère, on trouve plusieurs oocytes parfaitement constitués (Pl. X Fig. 14,oo). Au

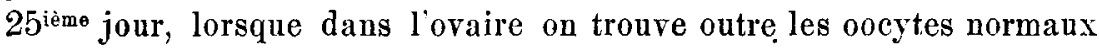
plusieurs abortés, il est impossible de distinguer l'ovaire régénéré de l'ovaire d'un individu normal pris dans la même époque.

Comme l'on voit la marche de la régénération de lovaire est analogue à l'évolution normale et les produits sexuels ne différent point morphologiquement des produits normaux.

Chez Pol. nigra l'intestin est déjà reconstitué en 7 jours et le ganglion nerveux avec les trones nerveux longitudinaux et les testicules en 10 jours. On observe à peu près la même chose chez les antres Triclades d'eau douce, mais tandis que chez Pol. nigra à la régénération de divers systèmes organiques suit presque immédiatement (de un à trois jours) la première différenciation de l'ovaire, 
chez Planaria polychroa selon Lang, Pl. lugubris selon Stevens, $P l$. torra et Dendrocoelum lacteum selon Schultz, on n'observe pas du tout la régénération de l'ovaire. Enfin, selon la constatation de A. Montr chez $\mathrm{Pl}$. torva, on observe la régénération des ovaires seulement en 7 mois!

De quoi dépend cette différence? Faudra-t-il admettre la propriété de la régénération chez $P o l$. nigra et la nier chez les autres espèces eitées, si proches par l'organisation de cette première? Ou faudra-t-il admettre la non réalisation des conditions favorables pour la régénération? Que devient-il alors des individus dépourvus de l'ovaire? Je suis d'opinion que la cause des résultats négatifs consiste précisément dans les conditions défavorables pour la régénération de l'ovaire.

La régénération des ovaires chez Pol. nigra se produit avec la même facilité, aussi bien au mois de Décembre qu'au mois de Mai, naturellement avec une plus grande vitesse à la température élévée, comme nous le verrons plus loin. Elle est donc complètement indépendente des saisons. La multiplication des ovogonies chez Pol. nigra est limitée; il y a done un nombre constant d'ovogonies et précisement 64 . Un certain nombre de celles-ci est aborté, de sorte que dans l'ovaire complètement régénéré on trouve de 56 à 60 ovogonies normales, comme j'ai pu m'assurer maintes fois dans mes préparations.

Jusqu'à ce stade je n'ai jamais trouvé des oocytes dans l'ovaire; et e'est seulement à partir d'ici que les orogonies entrent en évoIntion.

Vitellogène. Le développement ontogénétique du vitellogène est successif comme en le sait au développement du germigène. La même chose se vérifie aussi dans la régénération.

Chez Pol. nigra les premières cellules vitellines apparaissent lorsque le germigène est entouré déjà de tunica propria et contient au moins 16 ovogonies; et précisément dans quelques préparations, depuis 13 jours après l'opération et à la température de $14^{\circ} \grave{a} 16^{\circ} \mathrm{C}$. j'ai constaté le vitellogène contenant 6 cellules vitellines. Avant cette époque, malgré les très nombreuses expériences que j'ai faites, il m'étais impossible de trouver des traces de vitellogène. C'est le stade le moins érolu que j'ai pu constater dans mes préparations. Aux dépens de quoi régénère le vitellogène? Nous avons $\mathrm{vu}$, au commencement de ce châpitre, que la cellule de régénération qui 
a pris la voie germinale de la différenciation c. à d. la cellule-mère du germigène, en se divisant, donne lieu à deux cellules. Celles-ci au debut égales, augmentent ensuite inégalement de volume. On observe cela constamment. Nous avons vu en outre que dans toutes les préparations c'est la cellule la plus grande qui entre la première en évolution, tandis que l'autre reste indivisée. Lorsque le germigène est constitué de 3 ovogonies (Pl. X Fig. 11,ov), une des ovogonies diffère des autres par la grandeur, la forme et en partie par la granulation du protoplasma. Plus tard cette cellule manque.

Or a quoi bon cette constante différenciation précoce des deux cellules primaires, ainsi formées, et leur position déterminée?

Bien que je n'aie pas des données plus précises, je crois que cette différenciation précoce tient à la fonction diverse des deux premières cellules de l'ovaire. Je pense par conséquence qu'il faut admettre l'origine commune dans la régénération des gonades femelles (germigène et vitellogène) chez Pol. nigra et précisément qu'une cellule de régénération différenciée, en se divisant, donne lien à deux cellules filles, l'une desquelles constitue la cellule-mère du germigène et l'autre la cellule-mère du vitellogène. Il me manque malheureusement l'observation directe des premiers stades évolutifs de cette cellule. Le stade le moins évolu que j'ai pu constater e'est le vitellogène entouré de sa membrane et contenant 6 cellules vitellines. Leurs protoplasmas sont fortement granuleux; les noyaux sont généralement ovales et sans aucune structure et se colorent très fortement en brun foncé par la double coloration de l'orange G et de l'hématéine I A.

Ces cellules ainsi formées se multiplient et déjà en 21 jours après l'opération dans quelques préparations $j$ 'ai trouvé le vitellogène complètement régénéré avec un grand nombre de cellules (PI. X Fig. 17 à 18, $v i t$ ). Les cellules vitellines jeunes, c. à d. qui ne contiennent encore les goutelettes nutritives jaune-clair, ressemblent fortement, à un examen superficiel, aux jeunes ovogonies; mais un examen plus profond montre immédiatement outre les différences citées la présence de nucléole dans les ovogonies tandis que les noyaux des cellules vitellines ne montrent aucune structure.

Chez les individus régénérés le vitellogène ne prend que très rarement le développement normal. Dans les diverses préparations des individus expérimentés dans les conditions normales et identiques de riche nutrition et de jeûne le vitellogène se trouve soit très peu développé, soit presque rudimentaire soit complètement absent. Dans 
quelques rares cas seulement le vitellogène est normalement développé et cela uniquement chez les individus qui ont été bien nourris après la régénération de tous les organes, c. à $d$. en 15 jours après l'opération.

Chez les individus qui ont subi le jeûne prolongé on ne trouve pas de traces du vitellogène. On voit donc qu'il existe des nombreuses irrégularités, dont la nature doit être recherchée, non exclusivement dans la nutrition, du moment que tous les individus d'une même catégorie se trouraient dans les conditions identiques de nutrition. C'est à cause de cela qu'il m'était impossible de suivre graduellement la régénération du vitẹllogène.

La régénération du vitellogène comme nous l'avons décrite s'accomplit dans les conditions identiques à la régénération de l'ovaire, et la régénération du vitellogène gauche et droit se produit contemporanément. Nous pouvons dès maintenant dire qu'il nous était impossible de trouver une influence quelconque de la température sur la régénération du vitellogène, tandis que comme nous le verrons plus loin la température est un des agents les plus puissants sur la régénération du germigène. Il s'en suit clairement de ce qu'il a été dit plus haut, que les vitellogènes peuvent être régénérés.

Ainsi nous avons démontré la possibilité de la régénération des ovaires (germigène et vitellogène) et nous avons pu suivre l'histogénèse de ces organes.

Cette régénération dans les conditions normales donne lieu, comme nous avons vu, aux éléments sexuels normaux, les éléments qui étant fécondés donnent lieu à la formation et à la déposition des cocons. En effet une vingtaine d'individus au $45^{\text {ième }}$ jour après l'opération, c. à d. complètement régénérés, ont été separés et mis dans une grande culture. Les animaux ont été alimentés avec les lombriciens et le liquide cultural était changé tous les 3 ou 5 jours. En deux semaines les animaux régénérés ont acquis une grandeur presque normale, e. à d. de 7 à $8 \mathrm{~mm}$. et déjà 6 semaines plus tard à la suite d'une regulière alimentation et précisement le 15 Mars ils ont déposé les cocons. Cela nous démontre d'une manière évidente que les éléments germinaux régénérés sont aussi physiologiquement normaux et aptes à la fécondation.

La régénération des ovaires et la grandeur des tronçons. Nous avons vu que dans les conditions normales et à la température de $14^{\circ}$ à $16^{\circ} \mathrm{C}$. la première différenciation de l'ovaire se pro- 


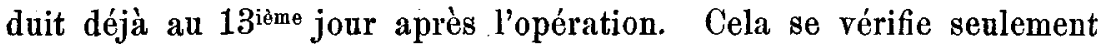
dans le cas lorsque la position des coupes est dans la région indiquée ce qu'implique la grandeur des tronçons non inférieure à $4 \mathrm{~mm}$. et dans la plus grande partie des cas non inférieure à $5 \mathrm{~mm}$. On sait que les animaux présentent une diverse poténtialité de régénération selon les divers niveaux des coupes. En effet Dribsch a demontré, que les surfaces des sections dirigées dans la direction orale des segments isolés de Tubularia, pris aux divers niveaux produisent les hydrantes, plus tard autant que plus aborale a été la position originaire des segments. Plus recemment Cerfontaine, Child, Torrey ont donné les autres exęmples du même phénomène. Chez les planaires les résultats ont été peu concordants. Ainsi Pl. maculata selon RANDOLPH coupée en huit morceaux est capable à régénérér chacun un individu arec la même rapidité. Selon Strevers chez $\mathrm{Pl}$. simplicissima et $\mathrm{Pl}$. morgani le cerveau et les yeux régénèrent avec la même rapidité dans les fragments prépharyngiens et postpharyngiens. Le pharynx pourtant régénère un peu plus rapidement dans les fragments prepharyngiens que dans les postpharyngiens.

Chez Pol. nigra nous pouvons dire dès maintenant, que toutes les portions, jusqu'à un millimètre, régénèrent tous les systèmes organiques, sauf la gonade femelle, avec la même rapidité. Pour l'ovaire, comme il résulte de nombreuses expériences, la potentialité change selon la grandeur des tronçons. Dans ce but je me suis servi aussi d'individus de grandeur de 11,12 et $13 \mathrm{~mm}$. et ils ont été tenus dans des conditions initiales égales. Chez ces animaux j'ai coupé les tronçons de $9,8,7,6,5$ et $4 \mathrm{~mm}$. destinés à régénérer deux pour chaque catégorie et ils ont été tenus dans des conditions identiques. Déjà an $11^{\text {ième }}$ jour à la température de $14^{\circ}$ à $16^{\circ} \mathrm{C}$. et à la lumière du jour tous les individus complètement régénérés montrent l'ébauche de la gonade femelle presque également développée. Les différences consistent dans le nombre des ovogonies qui oscille entre une seule et quatre. C'est le maximum d'ovogonies que l'on observe dans ces conditions-ci. Cela nous montre d'une manière évidente, que la potentialité de régénération de tous les organes sans exception ne varie pas selon la grandeur des tronęons et elle est égale pour tous ceux qui sont compris entre les limites de 9 à $5 \mathrm{~mm}$. Les tronçons de $4 \mathrm{~mm}$. ont la potentialité inférieure seulement en ce qui concerne la régénération des ovaires. Pour tous les autres organes, y compris les testicules, la potentialité de régénération ne change pas. En effet, en 11 jours dans une expérience faite sur 6 individus, deux 
examinés montrent tous les organes complètement développés, comme les tronçons de $9 \mathrm{~mm}$., mais on n'observe pas d'ovaires. Même en 12 jours, dans deux préparations examinées par moi, je n'ai pas trouvé d'ovaires. C'est seulement en 15 jours que j'ai constaté dans un cas une et dans l'autre quatre ovogonies dans la position normale. Dans une autre expérience les tronçons à régénérer ont mesuré de 3 à $4 \mathrm{~mm}$. et tandis que tous les organes ont été tout à fait régénérés en 15 jours les ovaires ont manqué complètement. En 18 jours dans deux individus seulement, j'ai trouvé la première ébauche de l'ovaire; dans tous les autres cas même en 21 jours il n'y avait pas de traces de la gonade femelle.

Les tronçons plus caudaux encore, et précisement de trois, deux et un $\mathrm{mm}$. en 11 jours, montrent tous les organes complètement développés, tandis que il n'y a pas de traces des ovaires. Cela nous montre d'une manière évidente que les tronçons de 3 à $1 \mathrm{~mm}$., tout en conservant la capacité de régénérer tous les systèmes organiques, perdent la potentialité de régénérer les ovaires.

Nous ponvons done distinguer trois zones de potentialité différentes pour la régénération' des ovaires chez Pol.nigra: la première de la potentialité maximum, qui est égale pour tous les tronçons du corps, compris entre 9 et $5 \mathrm{~mm}$; la deuxième, très limitée, de la potentialité minimum jusqu'à $4 \mathrm{~mm}$., ou un peu moins; et la troisième négative.

Il en résulte qu'il existe une limite inférieure de la potentialité nécessaire pour la régénération des ovaires. Tandis que la régénération du ganglion nerveux, de l'intestin, et des testicules s'accomplit , indépendamment de la grandeur du tronçons, c. à d. avec la potentialité toujours ou presque toujours égale, la régénération des ovaires demande un dégré de potentialité supérieur et pent-être spécifique d'une grandeur déterminée du tronçon. Et e'est pourquoi pendant les régénérations répétées, $P o l$. nigra qui ont mesuré $3 \mathrm{~mm}$. ou moins, n'ont jamais régénéré les ovaires, tandis qu'ils ont régénéré avec la plus grande facilité tous les autres organes.

Le tableau qui suit indique le rapport de la régénération avec la grandeur des tronçons du Pol. nigra.

Je considère comme le limite inférieur nécessaire pour la régénération des ovaires chez Pol. nigra la grandeur du tronçon de $4 \mathrm{~mm}$. Les tronçons un peu inférieurs à $4 \mathrm{~mm}$. seulement dans les

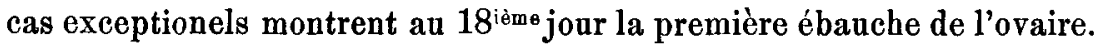
La lumière, la température élévée et les agents chimiques accéléra- 
Tableau I.

\begin{tabular}{|c|c|c|c|}
\hline \multirow{3}{*}{$\begin{array}{c}\text { Grandeur } \\
\text { des } \\
\text { tronçons }\end{array}$} & \multicolumn{3}{|c|}{ Temperature $\begin{array}{l}\min .14^{\circ} \\
\max .16^{\circ}\end{array}$} \\
\hline & \multicolumn{3}{|c|}{ Rapidité $d \theta$ régénération des oyaires } \\
\hline & 11 jours & 15 jours & 15 jours \\
\hline $9 \mathrm{~mm}$. & $\begin{array}{l}\text { Tons les organes. } \\
\text { L'ovaire uni- ou bicellulaire. }\end{array}$ & & \\
\hline $8 \dot{\mathrm{a}} 5 \mathrm{~mm}$. & id. & & \\
\hline $4 \mathrm{~mm}$. & $\begin{array}{l}\text { Tous les organes régénérés. } \\
\text { Manquent les ovaires. }\end{array}$ & $\begin{array}{l}\text { L'ovaire bi- ou tétra- } \\
\text { cellulaire. }\end{array}$ & \\
\hline 4-3 mm. & & & $\begin{array}{l}\text { Rarement } 1 \dot{a} 3 \\
\text { ovogonies. }\end{array}$ \\
\hline $3 \mathrm{~mm}$. & 'Tous les organes. & & \\
\hline $\begin{array}{l}2 \mathrm{~mm} . \\
1 \mathrm{~mm} .\end{array}$ & Manquent les ovaires. & & \\
\hline
\end{tabular}

teurs de la régénération sont sans aucune action sur les tronçons de $3 \mathrm{~mm}$. Les nombreuses expériences simultanées m'ont toujours confirmé ces résultats.

Nous verrons plus loin que la grandeur des tronçons jone un rôle très important dans la multiple régénération des ovaires chez Pol. nigra. Nous allons passer maintenant voir l'influence de la nutrition et $d u$ jeûne prolongé sur la régénération des ovaires chez notre animal. Or, étant donné que les animaux opérés dans les premiers jours ne prennent pas de nourriture, il ést évident que le jeûne court est sans aucune influence sur la régénération des ovaires chez Pol.nigra, parce que toujours, sans exception, dans les conditions déjà expliquées et à la lumière, j'observais la première différenciation de l'ovaire en 11 jours. C'est après la régénération de tous les organes et précisement 15 jours après l'opération que les individus commencent à se nourrir. Les individus nourris autant que ceux qui sont tenus en disette après la régénération, montrent en 20 jours les ovaires complètement constitués. Il semble pourtant que dans le premier cas les oocytes ont une grandeur majeure. Dans beaucoup d'autres cas on n'observe pas de différences. Il s'en suit que l'alimentation n'est pas unagent indispensable pour la régénération des ovaires chez Pol. nigra. La même opinion a été émise par Morgan pour la régénération des autres systèmes organiques chez $\mathrm{Pl}$. lugubris. 
Berninger pourtant, en étudiant l'influence du jeûne et de la riche nourriture sur la vie des oocytes du $P l$. torva, conclut que le premier de ces agents a une action nuisible. Les animaux qui m'ont servi pour les expériences ont été nourris régulièrement tous les 3 ou 5 jours avant l'opération et régénèrent très facilement, comme nous avons vu, les ovaires. Les autres animaux au contraire ont été tenus en disette pendant 2 ou 3 semaines et, opérés, ils ont régénéré normalement les ovaires. Done même le jeûne prolongé pendant 2 ou 3 semaines est sans action sur la régénération des ovaires chez Pol. nigra, lorsqu'il ne reduit les animaux à une grandeur inférieure à celle qui permet d'obtenir les tronçons de la grandeur nécessaire pour la régénération de la gonade. Dans le cas contraire, aucun des agents favorisant la régénération n'a d'action sur la formation de l'ovaire, sauf celui pourtant qui exerce une influence sur la grandeur du corps: la nutrition. En ce sens done le jeûne est un agent nuisible, spécifique pour la régénération de la gonade en question, puisque tous les autres organes peuvent être régénérés comme nous le savons, même par des individus de $1 \mathrm{~mm}$. Aussitôt que la grandeur des animaux augmente jusqu'à la grandeur nécessaire, et cela évidemment au moyen de riche nourriture, on voit chez les individus régénérés apparaître les ovaires: C'est ce que nous pouvions constater un grand nombre de fois pendant cette recherche.

En conclusion nous pouvons dire que Polycelis nigra régénèrent toujours les ovaires aux dépens d'une cellule embryonnaire. Cette régénération s'accomplit dans les conditions normales et à la température de $14^{\circ}$ à $16^{\circ} \mathrm{C}$. en 9 jours. Elle est indépendente des saisons. Elle s'accomplit avec la même facilité indépendamment de la nutrition, tant chez les individus grands, que chez les petits. Les animaux plus petits de $4 \mathrm{~mm}$. perdent la potentialité à régénérer les ovaires, tout en conservant la pleine potentialité pour la régénération des autres organes.

\section{L'influence de la lumière.}

Depuis les observations de Loeb et autres il est établi que la lumière exerce une action assez importante sur le développement des organismes. L'étude de cet agent a été porté aussi dans le domaine de la régénération, mais les résultats obtenus sont très peu concordants. Ainsi, selon Goldfarb, la lumière a une forte action accélératrice sur la régénération du Eudendrium racemosum. Peebles au 
contraire, démontre que la lumière et l'obscurité sont sans aucune action sur les hydres. Dernièrement Lava a étudié ce facteur sur la $\mathrm{Pl}$. polychroa et il amiva à la conclusion que la lumière exerce une action retardatrice sur la régénération. J'ai eu l'occasion d'étudier aussi ce facteur sur la régénération du Pol. nigra. Je me suis servi d'individus tenus dans les conditions initiales égales et les expériences ont été faites simultanément à la lumière du laboratoire et dans l'obscurité. Dans ce but 10 individus opérés ont été tenus à la lumière et 10 antres dans l'obscurité; les autres conditions ont été égales (la température et la grandeur des tronçons). En 7 jours après l'opération 2 individus de chaque milieu ont été fixés. L'examen microscopique montre chez les seconds le ganglion cérébral peu développé et dans la région néoformée on trouve encore une grande accumulation d'éléments parenchymateux (Pl. X Fig. 19, gn). Les cellules entourent complètement l'ébauche $d u$ ganglion en voie de formation. On n'observe pas de traces de la gonade femelle. Chez les animaux tenus à la lumière le ganglion est beaucoup mieux développé, mais on n'observe pas encore d'ovaires. En 9 jours après l'opération les différences dans le développement du ganglion sont encore accentuées. Chez les animaux tenus à la lumière le ganglion est presque complètement développé tandis que chez les autres il est encore entouré de plusieurs couches de cellules parenchymateuses. Dans tous les deux cas manquent les ovaires.

En 11 jours chez tous les animanx tenus à la lumière on observe la première différenciation de l'ovaire, tandis que chez ceux tenus dans l'obscurité il n'y en a pas de traces. Cette différence est constante et j'ai eu l'occasion de l'observer un grand nombre de fois. Le ganglion nervenx est presque complètement développé à cette époque chez les individus tenus dans l'obscurité. C'est en 13 jours seulement après l'opération qu'on observe l'ébauche de l'ovaire chez les animaux tenus en obscurité, dans le même temps - que les animaux tenus à la lumière ont l'ovaire bien constitué avec les nombreuses ovogonies. Il existe une correlation étroite entre la régénération du système nerveux et celle des ovaires et je crois que l'action de la lumière est indirecte sur la régénération de la gonade. A mon avis la lumière agit d'une manière accélératrice sur le ganglion, lequel chez les animaux à la lumière en 11 jours est déjà complètement développé tandis que chez les animaux tenus dans l'obscurité il arrive au terme de son développement seulement en 12 et même en 13 jours. Quant à l'influence de la lumière sur la régénération 
des yeux je dois dire, que dans les conditions normales ceux-ci sont complètement développés en 11 jours, tandis que chez les animaux tenus dans l'obscurité, on observe les yeux également développés seulement en 12 et même plusieurs fois en 15 jours.

Les différences individuelles sont fréquentes et il est nécessaire de faire des recherches quantitatives pour avoir le criterium exacte de l'action de cet agent. LANG en se basant sur un nombre trop petit d'individus arrive à une conclusion contraire à la mienne. Les résultats exposés plus haut ont été constatés aussi dans plusieurs expériences indirectes et je peux conclure que la lumière exerce une action accélératrice sur la régénération du Pol. nigra et particulièrement sur la régénération du système nerveux, des ovaires et des yeux. Nous verrons pourtant plus loin que la lumière accélère la régénération seulement lorsqu'elle agit dans des limites déterminés de température.

\section{L'influence de la température.}

Nous allons arrêter notre attention sur un autre agent, très important, de la régénération: la température. Il est évident a priori, que la température exerce une action générale sur la régénération. L'effet de la température sur la régénération a été étudié par plusieurs auteurs. Ainsi, Barfurth l'étudie sur les larves des Amphibiens et conclut que la régénération ne se produit pas à la température de $10^{\circ} \mathrm{C}$; - au contraire la régénération est accélérée à $28^{\circ} \mathrm{C}$. Pour les autres animaux, nous devons citer les expériences de Peebles qui a étudié la régénération des polypes d'eau donce. L'auteur a observé que la température de $26^{\circ}-27^{\circ}$ est très favorable pour la régénération. En augmentant encore la température jusqu'au $32^{\circ} \mathrm{C}$. le temps de la régénération diminue. Chez les planaires ( $P l$. torva) LiLlie et KNowlton ont observé, que la régénération a lieu déjà à la température de $3^{\circ} \mathrm{C}$. et que celle-ci est le limite inférieur nécessaire. Optimum de la température pour la régénération du $\mathrm{Pl}$. torva est de $29,7^{\circ} \mathrm{C}$. En commençant de $31^{\circ}$ jusqu'à $34^{\circ} \mathrm{C}$. la vitesse de la régénération diminue et les animaux meurent.

C'est uniquemènt pour déterminer l'influence de la température sur la régénération des ovaires chez Pol.nigra, que j'ai entrepris une série d'expériences à partir de la température variante entre $5^{\circ}$ et $7^{\circ} \mathrm{C}$. Dans ce but les animaux tenus dans les milieux naturels, ont été mis dans une chambre non échauffée dont la température a 
varié entre $5^{\circ}$ et $7^{\circ} \mathrm{C}$. pendant les mois d'hiver. Un certain nombre d'individus ont été tenus à la lumière du jour, les antres dans l'obscurité. En 11 jours après l'opération, chez les individus tenus à la lumière et dans l'obscurité, l'examen microscopique montre un état excessivement faible de développement du ganglion, comparable à celui qu'on observe en 6 jours chez les individus à la température entre $14^{\circ}$ et $16^{\circ} \mathrm{C}$. La fixation suivante des animaux a en lieu 15 jours après l'opération: le ganglion nerveux est encore incomplètement développé et on observe un bon nombre de cellules parenchymateuses autour du ganglion. On peut comparer cet état de développement à celui qu'on observe en 8 jours chez les animaux à la température de $14^{\circ}$ i $16^{\circ} \mathrm{C}$. C'est seulement en 19 ou 20 jours après l'opération qu'on observe le ganglion complètement développé chez tous les individus. Je pouvais constater en 21 jours et même plus tard et précisément en 25 la première différenciation de l'ovaire. Il y a lieu à constater les nombreuses irrégularités. Nous savons déjà à quel point de développement se trouve l'ovaire à la température normale. L'influence de la lumière $\dot{a}$ cette température est nulle. On observe, il est vrai, quelques rares indices qui parleraient en fareur de ce facteur, mais le manque de différences notables, positives, m'a induit à considèrer l'action de la lumière, à cette température, comme indifférente. L'ovaire complètement développé est observé dans ces conditions seulement en 35 ou 40 jours.

La régénération de l'ovaire à nne température de $9^{\circ}$ à $12^{\circ} \mathrm{C}$. s'accomplit beaucoup plus rapidement.

En effet déjà en 13 jours le ganglion est complètement développé et au $15^{\text {iéme }}$ on se trouve toujours en présence d'un ovaire uniou bicellulaire. Même ici il faut noter quelques cas d'irrégularité; les différences consistent dans le nombre des ovogonies. C'est la marche du phénomène à la lumière. Dans l'obscurité, au contraire, la régénération généralement est plus lente. En effet dans 6 sur 10 cas j'ai trouvé l'ovaire uni- ou bicellulaire seulement en 17 jours et même en $18^{\circ}$ après l'opération. L'ovaire complètement constitué dans tous les cas est observé en 25 -28 jours. Nous pouvons done considérer qu'à partir de cette température la lumière commence à exercer une influence sur la régénération. Par conséquence je considère la lumière comme un agent d'ordre secondaire, lequel entre en jeu, senlement, lorsqu'il est associé à une température déterminée.

Nous savons déjà quelle est la marche de la régénération à la température de $14^{\circ} \grave{a} 16^{\circ} \mathrm{C}$. A la lumière du jour le ganglion nerveux 
est complètement développé en 10 jours et la première différenciation de l'ovaire se constate 11 jours après l'opération, tandis que dans l'obscurité on l'observe en 13, quelques rares fois, en 12 jours. L'ovaire complètement développé est observé en 20 jours. Ici donc la lumière entre en jeu d'une manière constante avec un maximum de potentialité.

A une température variante entre $17^{\circ}$ et $19^{\circ} \mathrm{C}$. la régénération de l'ovaire, et en général de tous les systèmes organiques, s'accomplit plus rapidement. En effet en 9 jours après l'opération le ganglion nerveux est déjà complètement développé et en 11 jours j'ai trouvé dans l'ovaire de 8 à 12 ovogonies, tandis que chez les individus tenus dans l'obscurité l'ovaire également développé est observé généralement en 12 et même en 13 jours. Ici même j'ai trouvé plusieurs irrégularités, pourtant d'ordre secondaire. J'ai observé l'ovaire complètement constitué avec les oocytes, en 17 et même en 15 jours après l'opération. Les expériences faites à une température de $19^{\circ}$ à $21^{\circ} \mathrm{C}$. m'ont donné une plus rapide régénération. En effet, à cette température les premières ovogonies apparaissent en 7 jours après l'opération et en 11 et même en 10 jours on observe dans l'ovaire plusieurs oocytes. Dans l'obscurité il semble que la régénération se prodnit un peu plus lentement, mais les nombreux cas d'irrégularité m'induisent à considèrer comme nul cet agent à cette température. Aux températures supérieures la lumière et l'obscarité sont complètement indifférentes sur la régénération du Pol. nigra.

A la température du thermostate variante entre $23^{\circ}$ et $25^{\circ} \mathrm{C}$. la régénération s'accomplit encore plus rapidement. C'est déjà après 5 jours que je me suis trouvé en présence d'un ovaire contenant quatre, huit et même 16 ovogonies. A la température de $25^{\circ}$ à $28^{\circ}$ la régénération des ovaires s'accomplit dans le même temps comme à la température de $23^{\circ} \dot{a} 25^{\circ} \mathrm{C}$. Quelquefois pourtant 4 jours après l'opération, j'ai trouvé l'ovaire contenant de 4 à 8 ovogonies. Il me semble néanmoins que cette température n'est pas favorable en général à la régénération du Pol. nigra.

A une température de $29^{\circ}$ à $31^{\circ}$ en 4 jours après l'opération, on observe une forte accumulation d'éléments cellulaires déformés dans la partie opérée et en 6 jours généralement les animaux meurent. $A$ une température de $32^{\circ} \mathrm{C}$. les animaux opérés meurent en 24 heures. Les animaux normaux meurent à la température de $34^{\circ}$.

Je considère la température de $23^{\circ}$ à $25^{\circ}$ comme optimum pour la régénération des ovaires du Pol. nigra. 


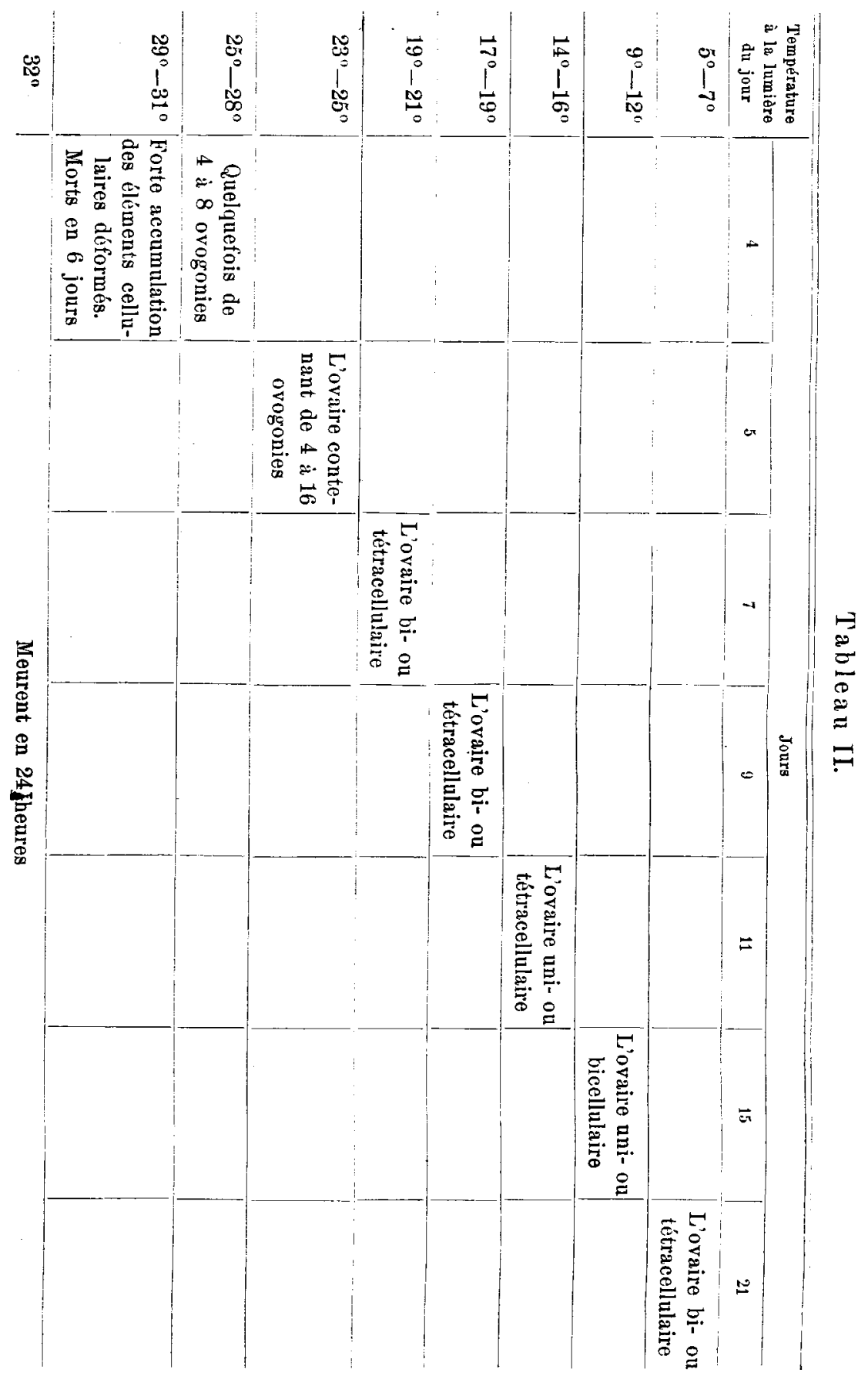


Il s'en suit, que la température a une action accélératrice bien accentnée sur le régénération et en dehors des limites déterminés elle compense l'action accélératrice de la lumière.

\section{L'influence du milieu salin.}

Dans ces dernières années on s'est occupé beaucoup de l'action du milieu salin sur les phénomènes biologiques et on a demontré l'extrème sensibilité des organismes à ces agents. Ainsi LoEb a démontré que le développement ontogénétique aussi que la régénération sont fortement influencés par le milieu salin. Ainsi pour Tubularia on observe le maximum du développement nou pas dans l'eau de mer normale $(3,8 \%)$ mais dans l'eau de mer un peu diluée $(2,2 \%)$. En augmentant la concentration le développement diminue et dans une concentration $5,1 \%$ le développement est nul. Les expériences de Herbst ont demontré aussi que pour la régénération il est nécessaire un certain degré de concentration saline. Steinmann en étudiant la régénération des Triclades marines arrive à la conclusion que les sels retardent la régénération de ces animanx.

Sur les animaux d'eau douce les expériences sont très peu nombreuses. Nous devons citer celles de Frazeur et Davenports qui ont demontré que même les faibles doses de $\mathrm{NaCl}$ diminuent la potentialité de la régénération chez les annélides.

Dernièrement STOCKand a étadié l'influence du milieu salin sur la régénération du Cassiopea xamachana et conclut que les sels ont une action très importante sur l'accélération on le retard des phénomènes de la régénération. Mongulis étudie aussi cet agent sur Lumbriculus et arrive à la même conclusion. L'action da milieu salin sur la régénération des planaires d'eau douce n'a pas été étudié, malgré même un très grand nombre de travaux.

J'ai entrepris une série d'expériences à fin de déterminer l'action spécifique de ce milieu sur la régénération de l'ovaire, aussi que sur la régénération générale du Pol. nigra.

J'ai expérimenté le $\mathrm{NaCl}$, le $\mathrm{CaCl}_{2}$ et le $\mathrm{FeCl}_{3}$ les sels les mieux connus dans leurs action sur les tissus; les composés fortement toxiques: le $\mathrm{HgCl}_{2}$ et $\mathrm{As}_{4} \mathrm{O}_{6}$. En outre j'ai expérimenté l'HCl et le $\mathrm{NaOH}$ pour déterminer si la régénération est favorisée par le milieu acide on alcalin.

Nous allons exposer les résultats détaillés obtenus avec chacun de ces composés. 
Tous les expériences ont été faites essentiellement à la lumière du jour, Les $\mathrm{NaCl}, \mathrm{CaCl}_{2}$ et $\mathrm{FeCl}_{3}$ aussi que le $\mathrm{HgCl}_{2}$ et $\mathrm{l}^{\prime} \mathrm{As}_{4} \mathrm{O}_{6}$ ont été expérimentés à la température de $14^{\circ}$ à $16^{\circ} \mathrm{C}$. Les autres températures sont indiquées à leur place.

\section{NaCl.}

L'action de ce chlorure est spécifique sur la régénération des ovaires du Pol. nigra. En effet, dans une concentration de $\frac{m}{12}$ où les planaires vivent très bien, la régénération de tous les organes, sauf la gonade femelle, s'accomplit normalement en 11 jours. C'est seulement en 15 jours, qu'il m'était possible de trouver l'ovaire uni- on bicellulaire à cette concentration de $\mathrm{NaCl}$. Aussi à une concentration $\frac{\mathrm{m}}{30}$ le $\mathrm{NaCl}$ a une action retardatrice. En effet 15 jours après l'opération j'ai trouré l'ovaire contenant le même nombre d'ovogonies que dans la concentration $\frac{\mathrm{m}}{12}$ tandis que l'on observe tous les antres organes complètement développés déjà en 11 jours. Quelquefois en 13 jours on observe déjà la cellule mère de l'ovaire, mais dans cette solution la multiplication des ovogonies est très lente. En 15 jours après l'opération on trouve généralement l'ovaire bicellulaire. Une seule fois sur 4 j'ai trouvé l'ovaire tétracellulaire. Nous savons à quel point arrive la multiplication des ovogonies dans les conditions normales. A la concentration $\frac{\mathrm{m}}{120}$ le $\mathrm{NaCl}$ est moins retardataire et en 15 jours j'ai trouvé l'ovaire contenant de 8 à 10 ovogonies; aussi à une concentration $\frac{\mathrm{m}}{600}$ la $\mathrm{NaCl}$ est peu favorable. A cette concentration en 15 jours on constate dans l'ovaire jusqu'a 16 ovogonies. Dans une solution $\frac{m}{1200}$ on constate la première différenciation de l'ovaire en 11 jours et en 15 après l'opération on trouve aussi 16 ovogonies; dans deux cas sur 6 j'ai trouvé seulement 8 ovogonies. Les différences entre ces deux dernières concentrations sont très faibles et il faut citer plusieurs cas d'irrégularités. A partir de la concentration de $\frac{\mathrm{m}}{12.000}$ le $\mathrm{NaCl}$ est complètement indifférent sur. la régénération du Pol. nigra.

Dans l'obscurité ce chlorure conserve ses propriétés retardatrices. 
Si l'on augmente la température $\left(23^{\circ}-25^{\circ} \mathrm{C}\right.$.) le $\mathrm{NaCl}$ a une action nuisible, surtout dans les concentrations de $\frac{\mathrm{m}}{12} \grave{\mathrm{a}} \frac{\mathrm{m}}{30}$. A une température de $19^{\circ}$ à $21^{\circ} \mathrm{C}$. le $\mathrm{NaCl}$ jusqu'à la concentration $\frac{\mathrm{m}}{1200}$ a une action retardatrice; dans les concentrations plus faibles il se montre indifférent, comme à la température de $14^{\circ} \grave{a} 16^{\circ} \mathrm{C}$. Il s'en suit que le $\mathrm{NaCl}$ exerce une action retardatrice sur la différenciation germinale des cellules embryonnaires, aussi que sur la multiplication des ovogonies.

STockard en étudiant l'influence de ee chlorure sur Cassiopea xamachana arrive aussi à la conclusion que le $\mathrm{NaCl}$ exerce une action retardatrice sur la régénération.

Il résulte de mes expériences que ce chlorure dans les concentrations faibles, même à $0,48 \%\left(\frac{\mathrm{m}}{1200}\right)$, a une action nuisible sur la régénération de l'ovaire. Cela nous démontre une grande sensibilité des ovogonies pour les doses faibles de ce chlorure.

\section{$\mathrm{CaCl}_{2}$.}

Ce chlorure, au contraire, est très favorable, à la régénération des ovaires du Pol. nigra.

A la concentration $\frac{\mathrm{m}}{30}$ pourtant tous les organes sont complètement développés en 11 jours, sauf les ovaires. Dans cette concentration donc le $\mathrm{CaCl}_{2}$ a une action retardatrice uniquement sur les ovaires. C'est seulement en 12 ou en 13 jours après l'opération, qu'il m'était possible de trouver l'ovaire uni- ou bicellulaire et en 15 jours je n'ai jamais trouvé plus de 4 ovogonies. Nous savons que les animaux dans les conditions normales possèdent à cette époque l'ovaire contenant jusqu'à 16 ovogonies. A partir de la concentration $\frac{\mathrm{m}}{60}$ le $\mathrm{CaCl}_{2}$ se montre accélérateur sur la régénération du Pol. nigra. En effet, en 11 jours après l'opération, l'ovaire est déjá muni de tunica propria et contient de 4 à 8 ovogonies, tandis que en 13 jours l'ovaire contient presque toujours 32 ovogonies. On observe le maximum de l'accélération chez les individus tenus dans une concentration $\frac{m}{90}$ de $\mathrm{CaCl}_{2}$. En effet, en 13 jours après l'opération, chez ces animaux l'ovaire contient normalement 64 ovo- 
gonies. Cette action de $\mathrm{CaCl}_{2}$ à la concentration $\frac{\mathrm{m}}{90}$ est constante. Les mêmes effets produit ce chlorure à la concentration $\frac{\mathrm{m}}{180}$. Dans tous les deux cas on observe aussi le vitellogène, mais rélativement peu développé.

Il s'en suit, que le $\mathrm{CaCl}_{2}$, dans ces concentrations à une action accélératrice sur la multiplication des ovogonies et exerce en outre une action accélératrice générale, parce que tous les organes sont déjà complètement développés en 9 jours après l'opération et l'ovaire très souvent contient de 2 à 4 ovogonies. Aussi dans les concentrations plus faibles encore ce chlorure accélère la régénération.

En effet à la concentration $\frac{\mathrm{m}}{900}$ en 13 jours l'ovaire montre de 32 à 40 ovogonies. Dans cette concentration ce chlorure accélère la multiplication des orogonies à un dégré inférieur des concentrations $\frac{m}{90}$ et $\frac{m}{180}$.

Dans les solutions de $\mathrm{CaCl}_{2}$ à la concentration $\frac{\mathrm{m}}{1800}$ la multiplication des ovogonies est encore accélérée. En effet en 13 jours, j'ai trouvé toujours au moins 24 ovogonies. A la concentration $\frac{\mathrm{m}}{18.000}$ j'ai trouvé dans la plus grande partie des cas de 16 à 24 ovogonies. Comme nous voyons les différences entre ces deux concentrations sont très peu claires.

A partir de la concentration $\frac{\mathrm{m}}{180.000}$ le $\mathrm{CaCl}_{2}$ se montre indifférent sur la régénération du Pol. nigra, aussi que sur la multiplication des ovogonies. Dans quelques cas, il est vrai, j'ai trouré l'ovaire contenant 20 orogonies, mais il faut l'attribuer aux variations individuelles, parce que généralement l'ovaire contient jusqu'à 16 ovogonies, comme dans les conditions normales. La lumière et l'obscurité sont sans influence lorsque les animaux sont stimulés par le $\mathrm{CaCl}_{2}$.

Selon Stockard le $\mathrm{CaCl}_{2}$ a une action négative sur la régénération de la Cassiopea xamachana. Il résulte clairement de mes expériences que ce chlorure a une action favorable accélératrice de la régénération du Pol. nigra, aussi que sur la multiplication des ovogonies. Je considère comme l'optimum de $\mathrm{CaCl}_{2}$ les concentrations de $\frac{\mathrm{m}}{90}$ à $\frac{\mathrm{m}}{180}$. 
$\mathrm{FeCl}_{3}$.

Ce chlorure, que j'avais expérimenté dans plusieurs concentrations, exerce une action assez prononcée sur la régénération du $\mathrm{Pol}$. nigra. A une concentration $\frac{\mathrm{m}}{300}$ le $\mathrm{FeCl}_{3}$ se montre indifférent pour la régénération. En effet, 11 jours après l'opération, on trouve l'ovaire uni- ou bicellulaire et en 13 jours l'ovaire contenant jusqu'à 16 ovogonies, comme dans les conditions normales. Au contraire, la solution $\frac{m}{600}$ de ce chlorure a une action accélératrice, bien évidente. En effet, ici, en 13 jours, dans l'ovaire complètement constitué on trouve généralement 24 ovogonies. Plus accentuée encore est l'influence exercée par la concentration $\frac{\mathrm{m}}{2400}$ de ce chlorure: en 13 jours on constate toujours de 32 à 46 ovogonies, comme il m'étais possible de m'assurer sur 6 individus expérimentés. C'est la concentration optimum de $\mathrm{FeCl}_{3}$ pour la régénération du Pol. nigra. Dans les concentrations plus faibles ce chlorure exerce encore un action accélératrice, mais à un dégré inférieur. Ainsi à la concentration $\frac{m}{6000}$ dans l'ovaire, en 13 jours, on trouve constamment de 24 à 32 ovogonies, tandis qu'à la concentration $\frac{\mathrm{m}}{24.000}$ dans le même temps l'ovaire contient de 16 à 24 ovogonies. Les individus de control dans 2 cas montrent en 13 jours 12 ovogonies, dans un senl j'ai trouvé 16 ovogonies.

Nous voyons done, qu'à une concentration aussi faible, ce chlorure accélère encore la multiplication des ovogonies. Le ganglion nerveux est complètement développé en 9 jours déjà, comme j'ai pu le constater anssi dans les concentrations $\frac{\mathrm{m}}{2400}$ et $\frac{\mathrm{m}}{6000}$. A partir de la concentration $\frac{m}{60.000}$ jusqu'à $\frac{m}{240.000}$ le $\mathrm{FeCl}_{3}$ a une action minime sur la régénération du ganglion, autant que sur la multiplication des ovogonies. Dans les limites de ces concentrations le ganglion nerveux se développe complètement en 10 jours, rarement en 9 et l'ovaire contient à cette epoque de 2 à 4 ovogonies. Les concentrations plus faibles sont sans influence sur la vitesse de la régénération du Pol. nigra. Il s'en suit que le $\mathrm{FeCl}_{3}$ a une action 
accélératrice sur la multiplication des ovogonies aussi que sur la régénération générale.

Tous ces chlorures exercent donc une incontestable influence sur la régénération du Pol. nigra. Le $\mathrm{NaCl}$ à une action spécifique retardatrice sur la différenciation de l'ovaire et la multiplication des ovogonies, le $\mathrm{CaCl}_{2}$ et le $\mathrm{FeCl}_{3}$ exercent une action accélératrice générale.

Dans une expérience comparative, faite simultanément avec les $\mathrm{NaCl}, \mathrm{CaCl}_{2}$ et $\mathrm{FeCl}_{3}$ à la température de $14^{\circ}$ à $16^{\circ} \mathrm{C}$. les résultats exposés plus haut ont été complètement confirmés. Dans une solution de $\mathrm{NaCl}$ á la concentration $\frac{\mathrm{m}}{60}$ tous les organes sont complètement développés déjà en 11 jours, sauf les ovaires, qui se différencient seulement en 13 jours après l'opération. Le $\mathrm{CaCl}_{2}$ à la même concentration des ions $\left(\frac{\mathrm{m}}{90}\right)$ accélère la régénération de l'ovaire au maximum. A une concentration $\frac{m}{120}$ de $\mathrm{NaCl}$ en 13 jours après l'opération les animaux montrent l'ovaire bi- ou tétracellulaire, tandis que dans les solutions isotoniques de $\mathrm{CaCl}_{2}$ tous les organes ont été développés déjà en 9 jours et en 13 dans l'ovaire j'ai trouvé de 48 à 60 ovogonies. Le $\mathrm{NaCl}$ à la concentration $\frac{\mathrm{m}}{600}$ retarde encore la régénération, parce que seulement en 13 jours j'ai trouvé l'ovaire biou tétracellulaire, dans les solutions isotoniques $\mathrm{de}^{\mathrm{CaCl}_{2}}$, j'ai trouvé jusqu'à 32 ovogonies. Aussi dans une concentration $\frac{\mathrm{m}}{1200}$ de $\mathrm{NaCl}$, 13 jours après l'operation, j'ai observé dans 2 cas sur 4 l'ovaire contenant 8 ovogonies, dans 2 autres cas il y avait de 4 à 8 ; dans le $\mathrm{CaCl}_{2}$ à la même concentration des ions en 13 jours, les animaux possèdent jusqu'à 24 ovogonies et, le $\mathrm{FeCl}_{3}$ à la même concentration favorise la multiplication des ovogonies au maximum.

Le $\mathrm{NaCl}$ à $\frac{\mathrm{m}}{12.000}$ est indifférent pour la régénération, tandisque le $\mathrm{CaCl}_{2}$ à la même concentration des ions $\left(\frac{\mathrm{m}}{18.000}\right)$ accélère encore la multiplication des ovogonies. En effet, j'ai trouvé chez les animaux dans ces conditions de 16 à 24 ovogonies. Dans les solutions isotoniques de $\mathrm{FeCl}_{3}\left(\frac{\mathrm{m}}{24.000}\right)$ j'ai trouvé en 13 jours de 16 à 20 ovogonies. 
Les animaux de control, dans deux cas, possèdent l'ovaire contenant 10 et 12 ovogonies; dans un seul cas j'en ai trouvé 16 .

Il résulte de ce que nous avons dit que l'action exercée par des solutions salines n'est pas uniquement due à la tension osmotique, parce que les solutions différentes, mais isotoniques, exercent une action complètement différente. Je crois que cette influence est due à la nature intime des solutions salines.

$$
\mathrm{As}_{4} \mathrm{O}_{6} .
$$

Une action particulièrement accélératrice exerce l'anhydride arsénieux sur la multiplication des ovogonies aussi que sur la régénération générale du Pol.nigra. Cette action se manifeste surtout dans les concentrations très faibles.

Chez les individus tenus dans les conditions normales le ganglion nerveux est complètement développé en 10 jours. En 11 j'ai trouvé l'ovaire contenant de 6 à 8 ovogonies. Chez les animaux tenus dans une solution de $\mathrm{As}_{4} \mathrm{O}_{6}$ à la concentration $\frac{\mathrm{m}}{12.000}$, le ganglion nerveux arrive au terme de son développement senlement en 11 jours et l'ovaire est bicellulaire. Dans un cas j'ai trouvé l'ovaire tétracellulaire. Il s'en suit, qu'à cette concentration $1^{\prime} \mathrm{As}_{4} \mathrm{O}_{6}$ a une action retardatrice générale. Une action presque égale exerce l'anhydride arsénienx à la concentration $\frac{\mathrm{m}}{120.000}$.

Au contraire, e'est dans la solution $\frac{\mathrm{m}}{1.200 .000} \overline{0}$, que l'As $\mathrm{A}_{4} \mathrm{O}_{6}$ commence à accélérer la régénération. En effet ici le ganglion nerveux régénère en 10 jours, comme dans les conditions normales, et en 11 jours j'ai trouvé dans un cas 15 ovogonies, dans deux autres 12. La multiplication des ovogonies est beaucoup plus accélérée dans nne concentration $\frac{\mathrm{m}}{12.000 .000}$. Ici, en effet, 11 jours après l'opération on trouve jusqu'à 48 ovogonies. Le ganglion nerveux se régénère en 8 jours. Dans une solution $\frac{\mathrm{m}}{24.000 .000}$ en 11 jours j'ai trouvé dans l'ovaire jusqu'à 64 ovogonies et j'ai observé le ganglion nerveux complètement développé en 8 jours. Dans une concentration $\frac{\mathrm{m}}{60.000 .000}$ on trouve toujours en 11 jours l'ovaire contenant plusieurs oocytes du premier ordre. On observe la même chose dans la con- 
centration $\frac{\mathrm{m}}{120.000 .000}$. Au contraire dans la solution de $\mathrm{l}^{\prime} \mathrm{As}_{4} \mathrm{O}_{6}$, ’̀ la concentration $\frac{\mathrm{m}}{240.000 .000}$, en 11 jours dans l'ovaire j'ai trouvé dans un cas 48 ovogonies, dans deux autres 32 et 38 . A la concentration $\frac{\mathrm{m}}{600.000 .000}$ le nombre des ovogonies était égal à celui qu'on observe dans les conditions normales.

A une température de $19^{\circ}$ à $21^{\circ} \mathrm{C}$. les solutions de $\frac{\mathrm{m}}{12.000} \grave{a}$ $\frac{\mathrm{m}}{1.200 .000}$ de $\mathrm{l}^{\prime} \mathrm{As}_{4} \mathrm{O}_{6}$ sont nuisibles ou retardatrices.

Il s'en suit que l'anhydride arsénieux aux doses fortes a une action retardatrice sur la régénération du Pol. nigra; dans les doses faibles a une action fortement accélératrice et dans les diluitions très fortes son action est nulle. Comme optimnm je considère les concentrations comprises entre $\frac{\mathrm{m}}{60.000 .000}$ et $\frac{\mathrm{m}}{120.00 \overline{0.000}}$.

\section{$\mathrm{HgCl}_{2}$.}

Le sublimé dans les concentrations même très faibles a une action fortement retardatrice.

Les animaux ne supportent pas une concentration de $\frac{\mathrm{m}}{480.000}$ de ce chlorure. Au contraire la concentration $\frac{\mathrm{m}}{960.000}$ est déjà favorable à la vie des animaux, mais fortement retardatrice à la régénération.

En effet, 11 jours après l'opération, à la température de $17^{\circ}$ à $19^{\circ} \mathrm{C}$. le ganglion nerveux est excessivement peu développé et naturellement il n'y a pas de traces de la gonade femelle. Dans les conditions normales les animaux possèdent l'ovaire contenant de $8 \dot{a}$ 12 ovogonies. La concentration $\frac{\mathrm{m}}{1.200 .000}$ est également défavorable pour la régénération.

Dans la concentration $\frac{\mathrm{m}}{6.000 .000} \mathrm{le} \mathrm{HgCl}_{2}$ a une action spécifique sur la différenciation germinale. En effet, 11 jours après l'opération le ganglion nerveux est complètement développé mais il n'y a non plus d'ovaires. La même chose est observée dans une concentration $\frac{\mathrm{m}}{12.000 .000}$. 
C'est seulement dans une concentration $\frac{\mathrm{m}}{60.000 .000}$ qu'on constate en 11 jours la présence de l'ovaire contenant de 2 à 4 ovogonies. Dans une concentration $\frac{\mathrm{m}}{120.000 .000}$ en 11 jours après l'opération j'ai trouvé dans l'ovaire complètement constitué 8 ovogonies dans un cas et 4 dans un antre.

Les diluitions plus fortes encore sont sans influence sur la régénération.

Nous allons étudier maintenant l'influence du milieu acide et alcalin sur la régénération da Pol. nigra, en nous arrêtant surtout sur la régénération des ovaires.

Ces expériences ont été faites à la température de $17^{\circ}$ à $19^{\circ} \mathrm{C}$. et à la lumière du jour. Nous savons, qu'à cette température la régénération du Pol. nigra est un peu accélérée.

\section{HCl.}

L'acide chlorydrique à une concentration de $0,01 \%$ se montre déjà favorable à la régénération. En effet, tous les systèmes organiques sont déjà complètement développés 11 jours après l'opération et l'ovaire contient normalement 16 ovogonies, tandis que chez les individus de control dans deux cas on observe l'ovaire avec 4 et 8 ovogonies.

Cela se vérifie à la lumière du jour, autant que dans l'obscurité et quelquefois, dans cette concentration, on trouve un nombre supérieur (24) d'ovogonies. Il s'en suit que l'HCl à cette concentration favorise la multiplication des ovogonies, tandis qu'il semble être indifférent sur la régénération des autres organes.

An contraire à une concentration $0,001 \%$ l'HCl a une action accélératrice aussi sur les autres organes. En effet, chez les animaux dans ces conditions, le ganglion nerveux se développe complètement en 8 jours et 11 jours après l'opération dans l'ovaire j'ai tronvé tonjours jusqu'à 32 ovogonies. L'HCl est beancoup plus favorable à une concentration de $0,0001 \%$. En effet, ici aussi le ganglion arrive au terme de son développement en 8 jours, mais en 11 jours j'ai observé toujours dans l'ovaire le nombre maximum des ovogonies $(60-64)$. Nous voyons done, que tandis que l'acide chlorydrique à la concentration $0,001 \%$ accélère la régénération du ganglion comme à la concentration $0,0001 \%$, dans le premier cas 
l'ovaire possède un nombre inférieur d'ovogonies. Cela nous montre d'une manière évidente, que l'action de ces solutions est spécifique sur la multiplication des ovogonies.

A une concentration $0,00001 \%$ l'HCl accélère encore la multiplication des ovogonies. En 11 jours, en effet, j'ai trouvé constamment de 24 à 32 ovogonies. Même à une concentration plus faible encore et précisement ì $0,000001 \%$ le nombre des ovogonies est supérieur à celui qu'on observe dans l'eau potable et précisement de 12 à 16 ovogonies.

Les solutions plus diluées encore se montrent indifférentes sur la régénération. Ces résultats se vérifient sans différences, à la lumière du jour, autant que dans l'obscurité. Il en résulte, que l'HCl, même aux doses excessivement faibles, a une action accélératrice sur la régénération du Pol. nigra.

Comme optimum, je considère la concentration $0,0001 \%$.

NaOH.

L'hydrate de $\mathrm{Na}$ dans plusieurs concentrations expérimentées se montre non favorable ou indifférent sur la régénération.

Avant tout les animaux ne supportent pas une solution à $0,01 \%$. Au contraire, la solution de $\mathrm{NaOH}$ à $0,001 \%$ est favorable à la vie des planaires, mais la régénération s'accomplit plus lentement que d'ordinaire. En effet, le ganglion arrive au terme de son développement seulement en 11 jours et alors on est en présence d'un ovaire bi- et très rarement tétracellulaire. Dans cette concentration donc le $\mathrm{NaOH}$ a une action retardatrice sur la régénération. Dans une solntion à $0,0001 \%$ tous les organes sont développés en 9 jours, mais les ovaires se différencient plus tard et 11 jours après l'opération j'ai trouvé dans deux cas 4 ovogonies dans un seul 8 .

A partir de la concentration $0,00001 \%$ le $\mathrm{NaOH}$ est indifférent autant sur la régénération générale, que sur la multiplication des orogonies.

Les individus de control, 11 jours après l'opération, possèdent dans un cas 16 ovogonies, dans denx autres 6 et 8 .

Une expérience comparative faite simultanément avec l'HCl et le $\mathrm{NaOH}$, montre que l'HCl à la concentration $0,01 \%$ accélère déjà la régénération de l'ovaire, tandis que le $\mathrm{NaOH}$ à la même concentration est nnisible à la vie des planaires. Or, dans le premier cas, en 11 jours j'ai trouvé de 12 à 16 ovogonies. 
A la concentration $0,001 \%$ l'HCl accélère assez fortement la multiplication des orogonies, tandis que le $\mathrm{NaOH}$ la retarde.

En effet, dans le premier cas, j'ai trouvé 32 ovogonies chez deux individus et 24 dans un seul, tandis que dans le $\mathrm{NaOH}$ à la même concentration, en 11 jours, j'ai trouvé l'ovaire tétracellulaire et dans un seul cas 8 ovogonies. L'HCl à la concentration $0,0001 \%$ accélère encore la régénération; en effet en 11 jours, j'ai trouvé dans l'ovaire de 56 à 62 ovogonies, tandis que le $\mathrm{NaOH}$ à la même concentration montre seulement de 4 à 8 ovogonies. Enfin, dans la solution de l'HCl à la concentration $0,00001 \%$, j'ai trouvé 28 ovogonies dans un cas et dans deux autres 32 . Le $\mathrm{NaOH}$ à la même concentration est tout à fait indifférent.

Il s'en suit que les expériences comparatives, sauf quelques petites irrégularités, m'ont confirmé pleinement les résultats obtenus séparément.

Morgulis, en étudiant l'influence de certaines solutions salines sur Lumbriculus, trouve que l'acidité a une action favorable sur la régénération. Les résultats obtenus par moi, m'induisent à partager l'opinion de cet auteur. Je dois en outre observer qu'un dégré rélativement faible de l'alcalinité du milieu $(0,0001 \%)$ a une action retardatrice sur la régénération et la multiplication des ovogonies chez Pol. nigra.

On peut se demander si les ovogonies soumises à l'action des agents chimiques accélérateurs n'étaient pas plus petites, que les ovogonies dans les conditions normales. Dans cette hypothèse ces agents accéléraient seulement la division précoce de la cellule, mais n'influenceraient guère sa nutrition, parce que les ovogonies plus nombreuses dans une unité de temps, seraient en même temps plus petites. Or, il résulte de nombreuses préparations que dans les cas de $\mathrm{FeCl}_{3}$ et $\mathrm{d}^{\prime} \mathrm{As}_{4} \mathrm{O}_{6}$ les ovogonies sont plus grandes que d'ordinaire. Dans les autres cas les différences ont été inappréciables.

Nous avons observé en outre que les solutions salines accélératrices n'agissent pas directement sur la différenciation de l'ovaire, c. à d. qu'elles ne sont pas capables de provoquer la différenciation de l'ovaire, avant la régénération du système nervenx. Tous ces substances agissent avant tout sur la régénération du ganglion. Dans mes très nombreuses préparations je n'ai jamais trouvé l'ovaire même à l'état unicellulaire avant la régénération du système nerveux. Cela 
nous induit à conclure que le système nerveux est une condition sine qua non pour la régénération de l'ovaire du Pol. nigra.

\section{La régénération répétée des ovaires.}

La potentialité de la régénération est-elle limitée?

Les cellules embryonnaires, après avoir contribué à la régénération complète de la partie manquante, perdent-elles la potentialité de régénérer une autre fois tous les organes? ou bien cette potentialité est-elle variable pour les divers organes, lors des régénérations successives et selon le nombre de ces régénérations?

C'est pour répondre à ces questions, que j'ai opéré les animaux qui ont subi la première régénération. Nous savons que tous les animanx régénérés dans les conditions normales déjà en 21 jours après l'opération possèdent l'ovaire contenant les nombreux oocytes.

Généralement dans ces conditions les animaux mésurent de 5 à $6 \mathrm{~mm}$. Chez ces régénérats, j'ai coupé les segments de $3 \mathrm{~mm}$. en les laissant régénérer dans les conditions normales et à la température de $17^{\circ}$ à $19^{\circ} \mathrm{C}$.

En 11 jours après l'opération les animaux ont régénéré de nouvean tous les organes, mais il n'y a pas de traces de la gonade femelle.

La seconde fixation des animaux avait lieu 20 jours après l'opération, mais ici non plus on n'observe les ovaires. En 35 jours après l'opération les animaux expérimentés ne possèdent pas de gonade femelle. En ce temps les animaux non nourris ont diminué considérablement de grandeur. J'ai pensé alors à augmenter leur grandeur en les nourrissant; et 15 jours plus tard, c. à d. 50 jours après l'opération denx individus présentaient les ovaires complètement bien régénérés avec les nombreuses ovogonies. Les animaux ainsi régénérés m'ont donné 365 sections de $10 \mu$ chacune, e. à d. la grandeur des animaux était de $3 \mathrm{~mm}$. 65 et si nous supposons une contraction de $0,5 \mathrm{~mm}$. due à la fixation et à l'inclusion, nous voyons, que les animaux ont surpassé les $4 \mathrm{~mm}$. Les animaux dépourvus d'ovaires m'ont donné toujours jusqu'à 250 sections.

Il s'en suit de ce que nous arons que pendant la deuxième régénération les segments inférieurs à $4 \mathrm{~mm}$. se comportent d'une manière identique comme pendant la première.

De l'autre coté j'ai choisi les animanx qui après la première régénération, bien nourris, ont mesuré $8 \mathrm{~mm}$. de longueur. Or, en 
les coupant de manière à laisser à régénérer les segments de $5 \mathrm{~mm}$, 11 jours déjà après l'opération, dans tous les exemplaires j'ai trouvé l'ovaire complètement régénéré, avec plasieurs ovogonies.

Donc même ici il existe un rapport étroit entre la potentialité de régénérer les ovaires et la grandeur du corps.

Il résulte de ce que nous avons dit, que la deuxième régénération complète se produit avec la même facilité que la première.

En même temps plusieurs autre individus de la première régénération ont été opérés en laissant à régénérer les segments de 2 et $1 \mathrm{~mm} .11$ jours après l'opération les animaux ont régénéré tous les organes, sauf les ovaires. Donc les segments plus petits conservent la parfaite potentialité de régénérer pour la deuxième fois tous les organes, mais ils ne régénèrent pas les ovaires.

Nous allons voir comment se comportent pendant la troisième régénération les régénérats complets (c. à $d$. avec les ovaires). Ces régénérats nourris sont arrivés à une grandeur de 6 à $7 \mathrm{~mm}$. Chez ceux-ci j'ai coupé des segments de 4 à $5 \mathrm{~mm}$. et en les laissant régénérer dans les conditions normales et déjà en 11 jours en fixant plusieurs de cenx-ci, j'ai trouvé chez tous sans exception les ovaires complètement développés avec les nombreuses ovogonies. Donc la troisième régénération des ovaires s'accomplit normalement comme les précédentes si les segments à régénérer ne sont pas inférieurs à $4 \mathrm{~mm}$.

Je pouvais obtenir avec cette méthode 5 régénérations complètes successives du Pol. nigra.

Les ovaires dans toutes les régénérations se forment aux dépens des cellules embryonnaires.

Nous avons vu que les animaux une fois régénérés et qui mésurent moins de $4 \mathrm{~mm}$. sont capables de régénérer tous les organes, sauf l'ovaire, arec une vitesse normale. Mais sont-ils capables de régénérer et arec la même rapidité plusieurs fois successivement la partie manquante? Cette question est assez intéressante pour nons, si l'on pense que la potentialité complessive de l'animal est déjà diminué, parce qu'il se tronve dépourvu de l'ovaire.

Les animanx dépourvus de l'ovaire après la deuxième régénération, lesquels nous pourons appeler individus smâles", ont été opérés pour la troisième fois. Les individus de grandeur de 3,2 et $1 \mathrm{~mm}$. de longueur ont régénéré tous les organes, sauf les ovaires, déjà 11 jours après l'opération; et la marche de la régénération y est identique à celle qu'on observe pendant la première régénération. 
J'abtins donc la troisième régénération des individus * mâles * du Pol. nigra. Ces animanx après une pauvre alimentation ont été opérés pour la quatrième régénération. Ici même, avec la vitesse égale, en 11 jours j'avais obtenu les individus normaux, mais dépourvus de l'ovaire. Avec cette méthode j'ai réussi à obtenir la $5^{\text {ième }}$ régénération du Pol. nigra »mâle .

Les animaux ainsi obtenus ont-ils perdu la potentialité de régénérer la gonade femelle?

Polycelis nigra "mâles * ont été nourris très richement pendant 21-25 jours; après quoi trois individus ont été fixés. Dans deux cas j'ai trouvé l'ovaire contenant 4 ovogonies, dans un il n'y en avait pas de traces.

Il s'en suit, que les animaux n'ont pas perdu la potentialité de régénérer l'ovaire, au moins dans ce court espace de temps.

L'aspect déformé des ovogonies, leur cytoplasme parfaitement homogène, les noyaux transparents m'induisent à considérer pourtant ces éléments comme anormaux.

Nous pourons done conclure, que Pol. nigra a une potentialité de régénération illimitée. La régénération répétée de l'ovaire se fait toujours aux dépens des cellules embryonnaires. Celles-ci par consequence ont une potentialité illimitée pour régénérer tous les organes, sauf les ovaires, indépendamment de la grandeur $d a$ corps et une potentialité limitée à une grandeur déterminée du corps pour la régénération de l'ovaire.

\section{Conclusions.}

Nous avons démontré que Polycelis nigra régénère les gonades femelles: le germigène et le vitellogène. Cette régénération s'accomplit aux dépens des cellules embryonnaires et il $\mathbf{y}$ a tout lieu de supposer que ces organes régénèrent tous les deux aux dépens d'une seule cellule, laquelle en se divisant donne l'origine à la cellule-mère du germigène et à la cellule-mère du vitellogène.

A mon avis, les cellules embryonnaires doivent être considérées comme les éléments intermédiaires entre les cellules somatiques et les cellules germinales pr. dites.

Nous avons vu en outre qu'il existe un rapport entre la régénération de l'ovaire et celui du système nerveux. Le germigène se développe toujours après la régénération complète du ganglion nerveux. 
Tous les auteurs qui se sont ocenpés de la régénération ont observé que les organes génitaux se développent très tardivement.

Chez Pol. nigra nous avons vu, au contraire, que l'ovaire se développe immédiatement après la régénération de tous les autres organes, et précisement déjà 11 jours après l'opération dans les conditions normales et à la température de $14^{\circ}$ à $16^{\circ} \mathrm{C}$, on est en présence de la cellule-mère de l'ovaire. Le germigène régénéré acquit toujours le développement normal. Le vitellogène au contraire seulement dans des rares cas ce développe comme chez les animaux normaux.

La régénération des gonades gauche et droite s'accomplit contemporainement.

La régénération des ovaires s'accomplit indépendamment de la nutrition. Les animaux opérés ne prennent pas la nourriture qu'après la régénération de tous les organes.

Les ovaires régénérés ne différent guère des ovaires normaux et les produits sexuels étant fécondés se développent normalement.

La rapidité de la régénération des ovaires est, entre certaines limites, indépendante de la grandeur et de la position des coupes. Tous les segments jusqu'à $5 \mathrm{~mm}$. régénèrent avec la même rapidité. Les tronçons de $4 \mathrm{~mm}$. régénèrent uniquement les ovaires un peu plus lentement. Au delà de $4 \mathrm{~mm}$. l'animal conserve la potentialité de régénérer tous les organes, mais il ne régénère pas les ovaires:

En augmentant la grandeur des tronçons les ovaires réapparaissent. Par conséquence il existe un rapport étroit entre la grandeur des tronçons et la potentialité de régénérer les ovaires.

Je n'ai pas des donnés exactes à fin de pouroir dire si les tronçons inférieurs à $4 \mathrm{~mm}$., nourris d'une manière convenable et maintenus toujours dans cette grandeur, régénèrent ou non les ovaires. Jè sais disposé pourtant à croire que ces animaux ne régénèrent plus la gonade femelle. Cette question sera traitée prochainement.

La régénération des ovaires est hautement stimulée par des agents externes. Ceux-ci ont une action générale ou spécifique, accélératrice et retardatrice. Ainsi la lumière a une action accélératrice incontestable sur la régénération générale du Pol. nigra, lorsqu'elle agit dans les limites déterminées de la température. Cette température, que je ponvais déterminer exactement est de $14^{\circ}$ à $16^{\circ} \mathrm{C}$. Au dessus et au dessous de ces limites l'action de la lumière est surfaite par un agent plus puissant, qui est la température.

L'obscurité au contraire, dans les mêmes limites de la température, retarde la régénération. 
Lorsque la lumière agit dans ces limites avec des autres agents (solutions salines), son action est manifeste ou non selon la potentialité de ces agents mêmes.

La température a une action très importante sur la régénération dn Pol. nigra. Son action est générale. L'optimum de la température que j'avais determiné est de $23^{\circ}$ à $25^{\circ} \mathrm{C}$. La température de $32^{\circ}$ est mortale pour les animaux opérés. Les animaux normaux meurent seulement à $34^{\circ} \mathrm{C}$.

A la température optimum et dans les conditions normales la régénération complète de l'ovaire s'accomplit en 9 jours.

Les animaux, ou mieux les tronçons inférieurs à $4 \mathrm{~mm}$. commè nous savons, sont incapables de régénérer les ovaires. Dans ces conditions nul agent n'a d'action sur la régénération des ovaires. Le milieu salin exerce une action générale ou spécifique sur la multiplication des ovogonies.

Les agents spécifiques aussi que les généraux ont une action accélératrice, retardatrice ou indifférente. Chaque solution saline montre un optimum de concentration pour la régénération, an deçà duquel la solution est nuisible ou retarde la régénération, au delà elle est indifférente. L'action des agents chimiques n'est pas due à la tension osmotique des sels, mais à la nature intime de ces agents.

Il résulte que le $\mathrm{NaCl}$ a une action retardatrice spécifique sur la multiplication des ovogonies. Tous les autres organes dans ce milieu se développent aree une rapidité normale.

Le $\mathrm{CaCl}_{2}$ au contraire a une action fortement accélératrice dans les concentrations optimums, c. à d. de $\frac{m}{90}$ à $\frac{m}{180}$ sur la régénération générale du Pol. nigra. Une action aussi accélératrice exerce le $\mathrm{FeCl}_{3}$ aux concentrations de $\frac{\mathrm{m}}{2400}$ à $\frac{\mathrm{m}}{6000}$. Son action est pourtant inférieure à l'action de $\mathrm{CaCl}_{2}$ dans les concentrations optimums.

Les substances fortement toxiques ont une action très différente sur la régénération. Ainsi le $\mathrm{HgCl}_{2}$, dans la plus grande partie des concentrations expérimentées, se montre nuisible ou retarde la régénération des ovaires. Son action est spécifique sur les ovogonies, parce que tous les autres organes, dans certaines limites des concentrations, se régénèrent normalement.

$\mathrm{L}^{\prime} \mathrm{As}_{4} \mathrm{O}_{6}$ au contraire a une action fortement accélératrice sur la régénération générale et sur la multiplication des ovogonies. En 
effet, à la température de $17^{\circ}$ à $19^{\circ} \mathrm{C}$. à la concentration $\frac{\mathrm{m}}{60.000 .000}$, la première différenciation de l'ovaire s'accomplit an 7ième jour et 11 jours après l'opération on trouve dans l'ovaire complètement régénéré les nombreux oocytes, tandis que dans les conditions normales, dans le même temps on trouve jusqu'à 16 ovogonies. La régénération des ovaires est favorisée par un certain dégré d'acidité. L'HCl à des concentrations faibles $(0,00001 \%)$ accélère fortement la multiplication des ovogonies, tandis qu'un dégré relativement faible d'alcalinité la retarde $(\mathrm{NaOH}$ à $0,0001 \%)$.

Polycelis nigra est capable de régénérer plusieurs fois successivement tous les organes, y compris les ovaires, si les tronçons ne sont pas inférieurs à $4 \mathrm{~mm}$.

Dans les cas contraires l'animal ne régénère pas uniquement des ovaires; tous les autres systèmes organiques régénèrent normalement. Dans ces conditions j'avais obtenu les 5 régénérations complètes successives du Pol. nigra.

Les tronçons inférieurs à $4 \mathrm{~mm}$. sont capables de régénérer tous les organes, sauf les ovaires.

La potentialité de régénération chez ces animaux ne diminue pas avec les régénérations successives. On obtient ainsi des individus aux organes sexuels mâles uniquement.

Ces >mâles* régénèrent avec la même facilité tous les organes. Je suis arrivé à 5 régénérations successives de Pol. nigra, dépourvue de la gonade femelle.

Dans ce période les animaux ne perdent pas définitivement la potentialité de régénérer les ovaires, mais celles-ci, il me semble, sont anormales.

Je crois que dans un élévage approprié et prolongé les animanx perdent effectivement la propriété de régénérer la gonade femelle.

Alors on aurait la véritable transformation d'un individu hermaphrodite en un individu unisexuel mâle. Pourrait-on aussi obtenir un individu femelle? Ce caractère acquis serait-il héréditaire? A cette question d'une si haute importance les recherches futares ne tarderont pas peut-être à repondre.

La régénération des ovaires se fait toujours aux dépens des cellales embryonnaires. Celles-ci ont alors une potentialité illimitée pour régénérer tons les organes, y compris les ovaires, indépendamment de la grandeur des tronçons, pourvu que ceux-ci ne soient pas inférienrs à $4 \mathrm{~mm}$. Au dessous de cette grandeur la potentialité des 
cellules embryonnaires est limitée uniquement à la régénération de tous les autres organes, exception faite des ovaires.

Modena, Institut de Zoologie, d'Anatomie et Physiologie comparées de l'Université, Juillet 1914.

\section{Zusammenfassung.}

Polycelis nigra regeneriert die Ovarien (Keimstoff und Eidotterstoff) auf Kosten einer Keimzelle. Die erste Differenzierung der Ovarien beginnt nach 11 Tagen, bei einer Temperatur von 14 bis $16^{\circ} \mathrm{C}$. Die Regeneration der Ovarien erreicht binnen 20 Tagen ihr Ende. Dieselbe ist von der Ernährung unabhängig. Es existiert eine nähere Abhängigkeit zwischen der Größe der Schnitte und der Potentialität der Regeneration der Ovarien. Alle Segmente bis zu einer Länge von $5 \mathrm{~mm}$ regenerieren die Ovarien mit gleicher Schnelligkeit. Die $4 \mathrm{~mm}$ langen Segmente regenerieren die Ovarien ein wenig langsamer; unter $4 \mathrm{~mm}$ regenerieren die Schnitte die weibliche Gonade nicht mehr. Das licht übt eine beşchleunigende Wirkung auf die Regeneration der Ovarien aus, wenn es zwischen den von der Temperatar bestimmten Schranken wirkt $\left(14\right.$ bis $16^{\circ} \mathrm{C}$.). Die Finsternis im Gegenteil verzögert die Regeneration zwischen denselben Temperaturschranken. Die beste Temperatur für die Regeneration der Ovarien ist 23 bis $25^{\circ} \mathrm{C}$. Die operierten Tiere sterben bei einer Temperatur von $32^{\circ} \mathrm{C}$. Salziges Milieu hat eine beschleanigende oder verzögernde Wirkang auf die Regeneration der Ovarien. $\mathrm{NaCl}$ hat eine spezifisch verzögernde Wirkung auf die geschlechtliche Differenziernng der Keimzellen. $\mathrm{CaCl}_{2}$ und $\mathrm{FeCl}_{3}$ üben eine beschleunigende Wirkung auf die Vermehrung der Ovogonien aus. $\mathrm{As}_{6}$ beschleunigt sehr die Regeneration. Ein gewisser Grad von Sauersein begünstigt die Regeneration der Ovarien. Der Alkaligehalt im Gegenteil hat einen verzögernden Einfluß. Wenn die Tiere nicht unter $4 \mathrm{~mm}$ Länge abgeschnitten werden, so behalten sie die Fähigkeit, mehrfach in der Nachfolge alle Organe zu regenerieren. Die bis $1 \mathrm{~mm}$ kürzeren Schnitte, d. h. der Ovarien entblößt, behalten die Fähigkeit, das Tier mehrmals zu regenerieren, jedoch ohne die weibliche Gonade. Die wiederholte Regeneration der Ovarien geschieht immer auf Kosten der Keimzellen.

Prof. Antonio Turolla.

\section{Bibliographie.}

BARD, L., La spécificité cellulaire. Scientia. Biologie No. 1. Paris.

Berninger, J., Einwirkung des Hungers auf Planarien. Zool. Jahrb. Allg. Teil. Bd. 30. 1911. S. 198.

Davydoff, C., Sur la régénération de l'extrémité postérieure chez les Némertiens. Bull. Ac. imp. Sc. St.-Pétersbourg. 1909. p. 301-311.

Driesch, H., Studien über das Regulationsvermögen der Organismen. 1. Von der regulativen Wachstums- und Differenzierungsfähigkeit der Tubularia. Arch. f. Entw.-Mech. Bd. 5. 1897. S. 389-718.

Studien ïber das Regulationsvermögen der Organismen. 5. Ergänzende Beobachtungen an Tubularia. Arch. f. Entw.-Mech. Bd. 11. Hft. 1. 1901. S. $185-206$. 
Driesch, H., Regenerierende Regenerate. Arch. f. Entw.-Mech. Bd. 21. 1906. S. $756-792$.

Flexner, L., The Regeneration of the Nervous System of Planaria torva and the Anatomy of the Nervous System of Double-Headed Forms. Journ. of Morphol. Vol. 14. 1898. p. 337-346.

Funrmann, M., Sur les phénomènes de la régénération chez les Annélides. Arch. Sc. Nat. Vol. 5. 1898. p. 478.

GoldFarb, A. J., Experimental Study of Light as a Factor in the Regeneration of Hydroids. Journ. of Exp. Zool. Vol. 3. 1906. p. 129-152.

Haliez, P., Régénération comparée chez les Polyclades et les Triclades. C. R. Ass. Fr. 28e session. 1899-1900. p. 270.

JANDA, VICTOR, Die Regeneration der Geschlechtsorgane bei Criodrilus lacuum. Arch. f. Entw.-Mech. Bd. 33. Hft. 3/4. S. 345-348.

JiJIMA, J., Untersuchungen über den Bau und die Entwicklungsgeschichte der Süßwasser-Dendrocoelen (Tricladen). Zeitschr. f. wiss. Zool. Bd. 40. Bft. 3. 1887. S. $359-467$.

KING, H. D., Regeneration in Asterias vulgaris. Arch. f. Entw.-Mech. Bd. 7. 1898. S. $351-363$.

- Further Studies on Regeneration in Asterias vulgaris. Arch. f. Entw.-Mech. Bd. 9. 1900. S. 727-737.

Konschelt, E., Regeneration und Transplantation. Jena, Fischer, 1907. $286 \mathrm{~S}$.

LANG, P., Über Regeneration bei Planarien. Arch. f. mikr. Anat. Bd. 79. 1. Abt. 1912. S. $361-426$.

Levi, G., Lesioni sperimentali sull' abbozzo urogenitale di larve di Anfibi e loro effetti sull' origine delle cellule sessuali. Arch. f. Entw.-Mech. Bd. 19. 1905. S. 295 .

Lillie, F. R., Some Note on Regeneration and Regulation in Planarians. Amer. Natur. Vol. 34. 1900. p. 173-177.

- Notes on Regeneration and Regulation in Planarians. Amer. Journ. of Physiol. Vol. 6. 1901. p. 129-141.

Lili.ie, F. R., and Knowlton, F. P., On the Effect of Temperature on the Development of Animals. Zool. Bull. Vol. 1. 1898.

LOEB, J., On the Influence of the Reaction of the Sea Water on the Regeneration and Growth of Tubularia. Univ. Calif. Publ. Vol.1. 1907. p. 139-147.

Lothrop, E. H., Über die Regenerationsvorgänge im Eierstocke. Dissert. Zürich 1890.

Michel, Aug., Recherches sur la régénération des Annélides. II. Régénération céphalique. C. R. Soc. Biol. Vol. 4. 1897. p. 336-355.

— Recherches sur la régénération des Annélides. IV. Vitesse de régénération. Ibid. p. 385-387.

Monti, A., La rigenerazione degli ovari nelle planarie. Archivio Zoologico. Vol. 6. 1912. p. 27-35.

Mon'TI, R., La rigenerazione nelle planarie marine. Mem. del R. Istituto Lombardo. Vol. 19. Fasc. 1. 1900. p. 13.

Morgan, Lilian V., Regeneration of Grafted Pieces of Planarians. Journ. of Exp. Zoöl. Vol. 3. 1906. p. 269-294.

Morgan, T. H., Growth and Regeneration in Planaria lugubris. Arch. f. Entw.Mech. Bd. 13. 1899. S. 179-212.

- Regeneration in Planarians. Arch. f. Entw.-Mech. Bd. 10. 1900. S. 58-119. 
Morgan, T. H., Experimental Studies of the Regeneration of Planaria maculata. Arch. f. Entw.-Mech. Bd. 7. 1898. S. 364-398.

Morqulis, S., Observations and Experiments on Regeneration in Lumbriculus. Journ. of Exp. Zoöl. Vol. 4. 1907. p. 549-574.

- Contribution to the Physiology of Regeneration. II. Experiments on Lumbricnlus. Arch. f. Entw.-Mech. Bd. 28. 1909. S. 396-439.

Nussbaum, J., Weitere Regenerationsstudien an den Polychaeten. Über die Regeneration von Nereis diversicolor (0. F. Müller). Zeitschr. f. wiss. Zool. Bd. 89. Hft. 1. 1908. S. 109-163.

- Beitrag zur Frage über die Abhängigkeit der Regeneration vom Nervensystem bei Nereis diversicolor. Arch. f. Entw.-Mech. Bd. 25. 1908. S. 32-64.

Peebles, Fr., Experimental Studies on Hydra. Arch. f. Entw.-Mech. Bd. 5. 1897. S. $794-819$.

- The Effect of Temperature on the Regeneration of Hydra.

Pugnat, A., Note sur la régénération expérimentale de l'ovaire. C. R. Soc. Biol. Vol. 52. 1900. p. 265-266.

RANDOLPH, H., Observations and Experiments on Regeneration in Planarians. Arch. f. Entw.-Mech. Bd. 5. 1898. S. 352-372.

Schultz, E., Aus dem Gebiet der Regeneration. Zeitschr. f. wiss. Zool. Bd. 66. 1899. S. $605-624$.

— Aus dem Gebiet der Regeneration. II. Über die Regeneration bei Turbellarien. Zeitschr. f. wiss. Zool. Bd. 72. 1902. S. 1-30.

Stevens, N. M., Notes on Regeneration in Planaria lugubris. Arch. f. Entw.Mech. Bd. 13. 1901. S. 396-409.

- A histological Stady of Regeneration in Planaria simplicissima, Pl. maculata and Pl. morganii. Arch. f. Entw.-Mech. Bd. 24. 1907. S. 350-373.

Streinmane, P., La régénération chez les planaires. Arch. des Sc. phys. et nat. Vol. 31. 1908. S. 532-552.

Stockard, Ch. R., Studies of Tissue Growth. I. An Experimental Study of the Rate of Regeneration in Cassiopea xamachana. Ann. Biol. 1908. p. 123.

Wilhelm, J., Tricladen. Fauna u. Flora des Golfes v. Neapel. 1909.

\section{Explication des planches IX et $X$.}

Toutes les figures ont été dessinées à la chambre claire ABBE.

Fixatif Zenker. Colorations: Nos 3 et 4 avec le carmin boracique et le vert de méthyle; les Nos 14 et 18 avec le carmin boracique et le bleu de toluidine. Toutes les autres ont été colorées avec l'orange $G$ et l'hématéine I A APAThy, $10 \mu$.

\section{Polycelis nigra [Ehrenb.].}

Fig. 1. Individn normal. Coupe frontale. ger germigène; vit vitellogène; ovd oviducte. $\times 34$.

Fig. 2. Individu normal. Coupe transversale. t.n.l trone nerveux longitudinal; $p g$ pigment; oo oocytes. $\times 327$.

Fig. 3. Individu régénéré. Coupe transversale. Première différenciation de l'ovaire. $\mathrm{cm}$ cellule-mère de l'ovaire. $\times 327$.

Fig. 4. Détail de la fig. 3. c.r cellules de régénération; c.s cellules de soutiens. $\times 950$. 
Fig. 5. La cellule-mère de l'ovaire d'un autre individu. $\times 950$.

Fig. 6. Individu régénéré. Stade bicellulaire jeune. Coupe transversale. $\times 327$.

Fig. 7. Individu régénéré. Stade bicellulaire plus avancé. ov.i ovogonie interne; ov.e ovogonie externe. Coupe transversale. $\times 950$.

Fig. 8. Ovogonie interne en mitose. Coupe transversale. $\times 327$.

Fig. 9. Détail de la fig. précédente. $\times 950$.

Fig. 10. Stade successif. Les trois premières ovogonies. $<327$.

Fig. 11. Détail de la fig. précédente. $\times 950$.

Fig. 12. Ovaire tétracellulaire. t.p tunica propria. Coupe transversale. $>327$.

Fig. 13. Ovaire régénéré avec nombreuses ovogonies. Coupe transversale. $>327$.

Fig. 14. Ovaire régénéré avec des oocytes. oo oocytes.

Fig. 15. Individu normal. Le vitellogène, vit. Coupe transversale. $\times 327$.

Fig. 16. Une cellule vitelline normale. $\times 950$.

Fig. 17. Vitellogène régénéré. Coupe transversale. $× 327$.

Fig. 18. Cellules vitellines jeunes régénérées. $\times 950$.

Fig. 19. Individu régénéré. L'ébauche du ganglion, gn. Coupe transversale. $\times 327$. 

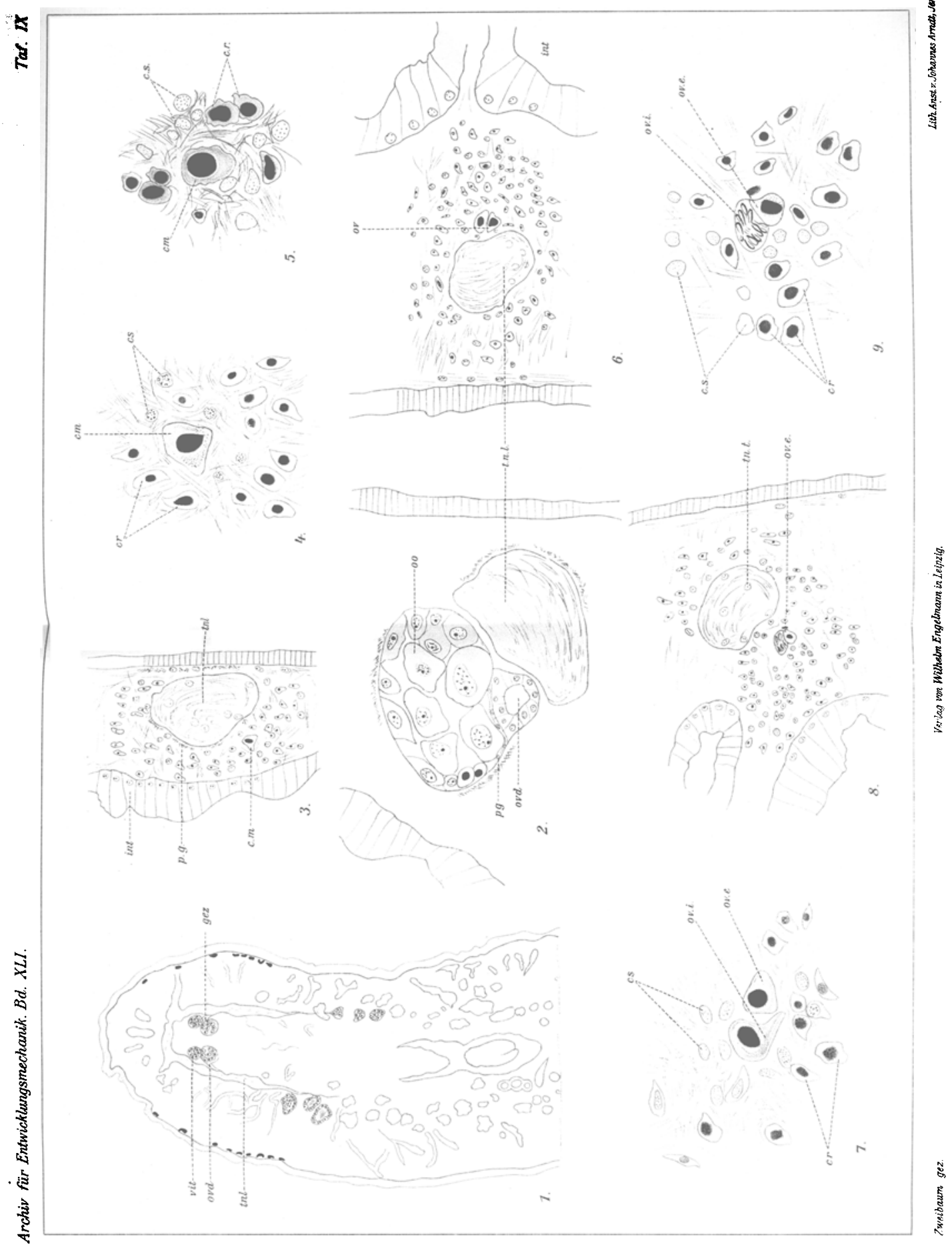


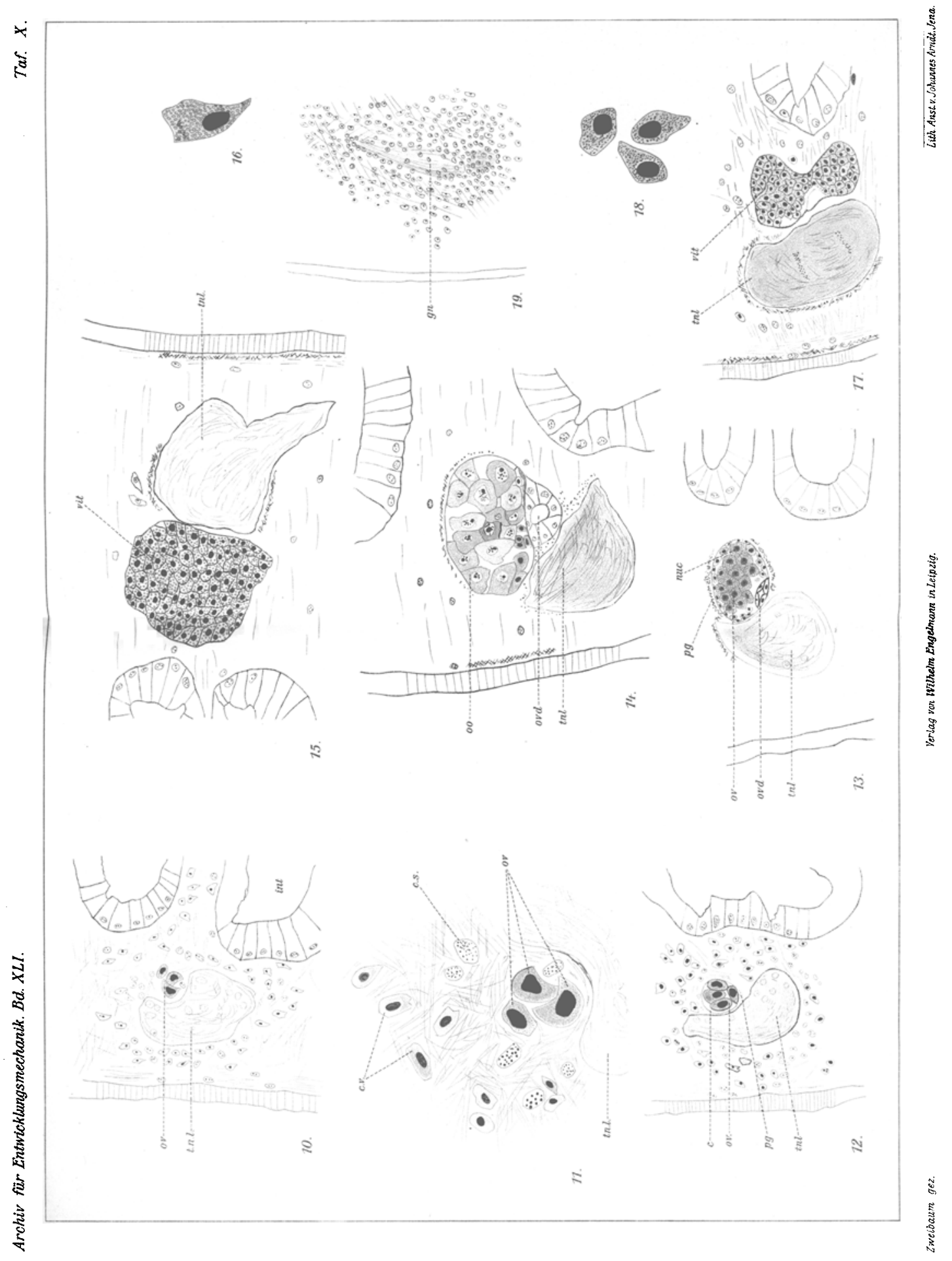

